$\angle$ Research Square

\title{
Mindfulness-Based Group Therapy as Support for Mental Health Concerns in Pregnancy: A Systematic Review
}

Amber Jenkins ( $\sim$ amberjenkins@msn.com)

Sunshine Coast University Hospital

Lauren Kearney

University of the Sunshine Coast

George Kendall

University of the Sunshine Coast

Lee Kannis-Dymand

University of the Sunshine Coast

\section{Research Article}

Keywords: pregnancy, mindfulness, group therapy, mental health, depression, anxiety

Posted Date: January 7th, 2022

DOl: https://doi.org/10.21203/rs.3.rs-1037571/v1

License: () (1) This work is licensed under a Creative Commons Attribution 4.0 International License. Read Full License 


\section{Abstract \\ Background}

Perinatal mental illness is prevalent and can be associated with poorer health outcomes for mother and fetus if untreated. Mindfulness is a contemporary approach to managing mental health concerns; however, little is known about the effectiveness of Mindfulness-based Interventions during pregnancy, especially within the context of peer support.

\section{Methods}

A systematic review was undertaken. Randomised controlled trials and quasi-experimental studies were included. All articles were critically appraised using the Critical Appraisal Skills Program checklist.

\section{Results}

Of the 2053 records initially identified, 21 studies met inclusion criteria. Studies demonstrated modest improvements in perinatal mental illness, particularly when interventions were adapted to meet the unique needs of women in the prenatal period. Comparison was difficult, due to high heterogeneity and methodological limitations. No studies explored peer support as a therapeutic mechanism and maternal-fetal bonding was not a measured in any studies.

\section{Conclusion}

Mindfulness-based group interventions designed to meet the needs of perinatal women require further research, with larger sample sizes, more rigorous methodology and greater demographic diversity required. Additionally, value could be afforded in exploration of how group support affects any change mechanisms within the participants and include maternal fetal bonding as a measured outcome.

\section{Introduction}

In Australia perinatal mental health concerns are common, and the impact can be significant, including suicide (1) and impaired parent-infant interaction (2). The most frequently reported perinatal mental health concerns are depression, anxiety and perinatal stress. Perinatal depression is defined as a major depressive episode during pregnancy or up to a year after childbirth (3) and is reported to have prevalence rates up to $20 \%$ during pregnancy and the first 3 months post-partum (4). It has been associated with cigarette smoking, alcohol consumption, weight gain, poor diet choices (5), disturbed sleep (6), chronic disease (7), preterm birth (5), and disordered maternal-fetal attachment, interaction and appropriate responsiveness $(2,14)$. Untreated maternal depression in pregnancy has also been found to increase the likelihood of depression in the child at 16 years by five times compared to those with mothers who did not have antenatal depression (8).

Perinatal anxiety has a bidirectional association with perinatal depression (9), and comorbidity is common (49.5\%). Diagnosis of an anxiety disorder in pregnancy can be complicated due to difficulties in distinguishing between normal and pathological worries as well as the normal physiological changes of pregnancy (10). Pregnancy anxiety may be described as generalised anxiety or pregnancy specific anxiety such as fear for health and well-being of self and baby, fear of childbirth and of the parenting role (11), characterised by excessive or uncontrolled worrying resulting in impaired functioning including fatigue, irritability, insomnia and concentration difficulties as well as physical symptoms such as rapid heart rate, muscular tension and breathlessness (10, 12). Pregnancy anxiety may also be compounded by dispositional anxious characteristics and be associated with insecure attachment, social isolation, cultural background, history of infertility and unplanned pregnancies (11). Anxiety and stress have been associated with increased nausea and vomiting, obstetric visits, and requests for elective caesarean section (13) as well as preterm birth; having a significant impact upon fetal neurological development (11). Anxiety in pregnancy has been negatively associated with the child's attention regulation, temperament, cognitive and motor development, emotional and behavioural problems as well as grey matter density (11). Excessive worry that continues in the post-natal period may also cause mothers to be less responsive and engaged with infants resulting in infant behavioural changes and negatively affecting mother-infant attachment $(10,14)$.

Also commonly reported, perinatal stress which is characterised by a major life event in pregnancy such as the death of a family member or chronic stress such as relationship and financial difficulties (11). These events may trigger a fetal programming response due to maternal stress hormones crossing through the placenta, thus impacting the future mental health of the child (2). Studies have found that up to $58 \%$ of women experience moderate stress through pregnancy (15) and $6 \%$ experience high levels of stress (11). Stress in pregnancy has been associated with increased likelihood of developing depressive symptoms (16). One study found that women who reported three or more stressful life events around the time of birth or social health issues had a greater than a five-fold increase in likelihood of depressive and anxiety symptoms (16). Whilst, pregnancy may be a time of great happiness for many women, for those experiencing depression, anxiety and/or stress another range of challenges are present.

Whilst awareness of perinatal mental illness has increased in recent decades, detection and treatment remain sub-optimal (17). Although Universal screening is recommended in Australia, numerous barriers persist (18). Maternity care is often fragmented with women seeing numerous care givers throughout their pregnancy despite the benefits of continuity of care giver being well documented (19)A high degree of variation of mental health assessment knowledge exists amongst midwives however, they are often not adequately supported with time and education (18). Lack of adequate referral pathways is also commonly reported (18). Identification of a mental health concern in pregnancy may provide no benefit if the woman chooses not to seek or receive further support and despite the serious nature of perinatal depression, studies have found between $50-80 \%$ of women do not seek help (15). The most common 
barriers to seeking help include denial and perceived stigma, combined with unacceptability of treatment options (18). Lack of finances, time, childcare and transportation have also been identified as challenges (18).

Contemporary perinatal mental health services need to address these barriers and delivered during pregnancy to mitigate impact upon the developing fetus and maternal health. One such activity aimed at addressing this are Mindfulness-Based Interventions (MBI). Mindfulness, derived from Buddhist traditions, has been defined as "paying attention in a particular way on purpose, in the present moment and non-judgmentally to the unfolding of experience moment by moment" (20, p. 4). Although mindfulness is an inherent capability, it may be enhanced through education and practice. Formal mindfulness programs have been developed for clinical treatments and therapy, including Mindfulness-Based Stress Reduction (MBSR) (21), Mindfulness-Based Cognitive Therapy (MBCT) (22) and Mindfulness-Based Childbirth and Parenting (MBCP) (23).

Mindfulness-based interventions have been effective in the treatment of mental health issues in the general population (24) and several studies have shown efficacy in the perinatal period, with improvements in anxiety, depression, worry, self-compassion and mindfulness $(25,26,27)$. The factors that have been identified as mechanisms of change in MBI's include cognitive change, self- management, relaxation, and acceptance (28). Mindfulness encourages an attitude of awareness and acceptance of physical sensations, feelings, and thoughts. Studies have found that mindfulness improves maternal autonomic nervous system function and has an advantageous effect on later infant socio-emotional behaviour as well as improving future maternal mental health (29). Parent-infant interaction may also improve as the parents' ability to be attentive to the infant is strengthened (25). Enhanced mindfulness may also assist with anxiety around childbirth by helping women to cope with the pain of childbirth through enhancing skills of remaining in the present moment and increasing ability to control focus and positive thought patterns (25). MBIs have since been used as a tool to reduce fear of childbirth (30), enhance breastfeeding efficacy (31) and improve parenting skills (32). Mindfulness-based skills may be taught in a group setting providing the added benefit of social support.

Perinatal Mindfulness-Based Interventions may be delivered individually or within a group setting. The group setting provides opportunity for peer support to be naturally embedded into this style of intervention. Peer Support has been well established in both mental health care (33) as well as in several areas of perinatal care including, childbirth preparation and breastfeeding (34). Peer Support may be simplistically defined as 'the giving of assistance and encouragement by an individual considered equal' (35 p. 323). Peer Support is based on the belief that people who have experienced adversity can offer useful support, encouragement and, hope to others experiencing similar situations (36).

The potential benefit of facilitated peer support in the context of Mindfulness-based support for pregnant women with mental health concerns is that support and knowledge from both healthcare professionals and the other participants are available. Social isolation is both a risk factor as well as a side effect of mental health concerns in the perinatal period (37). Social relationships have a significant impact on mental health through their influence on stress levels, depression, anxiety and well-being (38). However, some research suggests that in challenging times our usual social network may not respond positively, and support is received more readily from individuals who identify with and share common experiences (37). Studies in peer support groups in the perinatal period for depression report ongoing benefit from skills and knowledge acquired in the group as well as social support that extends beyond the completion of the group (37). Health centre-based peer support programs are also a more viable option to the public health system compared with individual based therapy (24). Psychological education and social support are key components in overcoming the denial and stigma associated with emotional health concerns and may help to develop self-efficacy in emotional health as well as overcome some of the help seeking barriers that pregnant women experience (10).

Despite the prevalence of perinatal depression, anxiety and stress and the emerging evidence to support MBI's in a group setting, no systematic reviews have been conducted which collate, synthesise and report the known attributes and benefits of MBI within the context of peer support as a therapeutic mechanism. Therefore we conducted a systematic review and critically appraised the literature relating to Mindfulness-based group interventions during pregnancy, and their effect upon perinatal depression, anxiety, stress and maternal-fetal bonding. An examination of the impact of peer support as a therapeutic mechanism in group-based mindfulness was also undertaken.

\section{Methods}

This review was conducted according to the preferred reporting items for systematic reviews and meta-analysis (PRISMA) statement (39). Published literature from 2008 - 2021 was reviewed through a systematic search of electronic databases CINAHL, Medline, Scopus and Psychinfo and Google Scholar. Search terms included "pregnancy", "pregnant", "antenatal", "perinatal", "maternal” in combination with "mindfulness", "mindful”, "group therapy", "peer support" and "mental health", "depression", anxiety", "stress".

\section{Eligibility Criteria}

Inclusion criteria were published in English in a peer-reviewed journal; involved pregnant participants who participated in a MBI group intervention including MBSR, MBCT, MBCP and their adaptations; and, measured pre- and post-intervention outcomes with validated mental health scoring tools. Quantitative and qualitative data studies were included, and no restriction was made on length, frequency or duration of MBI. Studies were excluded if the focus was on yoga, Acceptance and Commitment Therapy (ACT) or self-compassion, which all incorporate aspects of mindfulness practice, however, result in difficulties in interpreting the primary effect of mindfulness in the group environment. Studies were also excluded if the participants were recruited due to other health concerns such as obesity, diabetes, or severe fear of childbirth. Online interventions were also excluded as they are not attended in group format.

\section{Data extraction, synthesis and critical appraisal}

Data were extracted from full text articles and reviewed for comparison and exploration of homogeneity. Data included the intervention details and duration, method, recruitment and sample size, attrition, mental health measures and study results, a table of key concepts was produced (see Table 1). The Qualitative data was thematically analysed following extraction. Data were extracted from articles by two independent authors (AJ and KG) and evaluated using the 
appropriate tool from The Critical Appraisal Skills Program (CASP) and allocated a score (see Table 2 and 3). Studies were not excluded due to quality assessment; however, this is accounted for in later synthesis. 
Table 1

Characteristics of Studies

\begin{tabular}{|c|c|c|c|c|c|c|}
\hline Study & Intervention & Recruitment & Attrition & Measures & Result & CASP \\
\hline $\begin{array}{l}\text { Vieten and Astin } \\
\text { (2008) } \\
\text { USA } \\
\text { RCT with waitlist }\end{array}$ & $\begin{array}{l}\text { 2hr/week for 8- } \\
\text { week MBSR/ } \\
\text { MBCT including } \\
\text { some } \\
\text { psychological } \\
\text { education. } \\
\text { Readings and CD } \\
\text { for daily home } \\
\text { practice }\end{array}$ & $\begin{array}{l}n=31 \text { women } 12-30 \\
\text { weeks with history of } \\
\text { 'Mood concerns' } \\
13 \text { to intervention, } 18 \text { to } \\
\text { control }\end{array}$ & $\begin{array}{l}31 / 34 \\
\text { completed. } \\
\text { Mean } \\
\text { attendance } \\
-7.2 \text { sessions }\end{array}$ & $\begin{array}{l}\text { PSS } \\
\text { MAAS } \\
\text { STAI } \\
\text { CES-D } \\
\text { PANAS-X }\end{array}$ & $\begin{array}{l}\text { Decreased anxiety and depression, } \\
\text { not lasting postnatally }\end{array}$ & 10 \\
\hline $\begin{array}{l}\text { Duncan and } \\
\text { Bardacke (2009) } \\
\text { USA } \\
\text { Single group pre - } \\
\text { post intervention } \\
\text { Mixed methods }\end{array}$ & $\begin{array}{l}\text { Adapted MBSR } \\
\text { focusing on } \\
\text { Childbirth } \\
\text { education } \\
\text { Homework - } 30 \\
\text { Min/ day } 6 \text { days/ } \\
\text { week }\end{array}$ & $\begin{array}{l}n=27 \text { women in third } \\
\text { trimester and partners }\end{array}$ & $\begin{array}{l}\text { Mean } \\
\text { attendance - } \\
8.3 \text { sessions }\end{array}$ & $\begin{array}{l}\text { CES-D } \\
\text { DES } \\
\text { WOC } \\
\text { FFMQ } \\
\text { PSS } \\
\text { PAS } \\
\text { PANAS }\end{array}$ & $\begin{array}{l}\text { Significant improvements in stress } \\
\text { and coping into the post-natal period } \\
\text { Decrease in pregnancy anxiety and } \\
\text { stress } \\
\text { Increase in mindfulness } \\
\text { Modest improvement in positive } \\
\text { affect }\end{array}$ & 9 \\
\hline $\begin{array}{l}\text { Dunn et al (2012) } \\
\text { Australia } \\
\text { Pre and post } \\
\text { intervention mixed } \\
\text { methods study } \\
\text { with control group }\end{array}$ & $\begin{array}{l}8 \text { weeks } \\
\text { Adapted MBCT } \\
\text { focus on } \\
\text { depression }\end{array}$ & $\begin{array}{l}n=10 \text { pregnant women } \\
12-28 \text { weeks }\end{array}$ & $\begin{array}{l}10 / 11 \\
\text { Reported high } \\
\text { attrition rate }\end{array}$ & $\begin{array}{l}\text { DASS-21 } \\
\text { EPDS } \\
\text { SCS } \\
\text { MAAS }\end{array}$ & $\begin{array}{l}75 \% \text { reported decreased stress-related } \\
67 \% \text { improved self-compassion } \\
50 \% \text { reported improved depression }\end{array}$ & 6 \\
\hline $\begin{array}{l}\text { Goodman et al } \\
\text { (2013) } \\
\text { USA } \\
\text { Single group pre- } \\
\text { post intervention } \\
\text { with qualitative } \\
\text { interview }\end{array}$ & $\begin{array}{l}8 \text { weeks } \\
\text { 2hr/week } \\
\text { Adapted MBCT } \\
\text { with focus on } \\
\text { anxiety } \\
\text { Coping with } \\
\text { Anxiety through } \\
\text { Living Mindfully } \\
\text { (CALM) } \\
\text { Daily home } \\
\text { practice }\end{array}$ & $\begin{array}{l}n=24 \text { pregnant women } \\
\text { up to } 24 \text { weeks with } \\
\text { elevated anxiety and } \\
\text { moderate depressive } \\
\text { symptoms }\end{array}$ & $\begin{array}{l}23 / 24 \\
\text { completed } \\
\text { Mean } \\
\text { attendance - } \\
6.96 \text { sessions }\end{array}$ & $\begin{array}{l}\text { GAD-7 } \\
\text { BAI } \\
\text { PHQ-9 } \\
\text { BDI-II } \\
\text { MAAS } \\
\text { MINI } \\
\text { SCS } \\
\text { PSWQ }\end{array}$ & $\begin{array}{l}\text { Significant improvement in Anxiety, } \\
\text { worry and self-compassion }\end{array}$ & 10 \\
\hline $\begin{array}{l}\text { Guardino et al } \\
(2013) \\
\text { USA } \\
\text { RCT with } \\
\text { controlled reading } \\
\text { group }\end{array}$ & $\begin{array}{l}\text { 6-week } 2 \mathrm{hr} / \text { week } \\
\text { Mindfulness } \\
\text { Awareness } \\
\text { Practices Program } \\
\text { (MAPS) - } \\
\text { program designed } \\
\text { for general } \\
\text { community } \\
\text { CD and diaries of } \\
\text { home practice }\end{array}$ & $\begin{array}{l}n=47 \text { pregnant women } \\
\text { between } 10-25 \text { weeks } \\
\text { with elevated stress and } \\
\text { anxiety }\end{array}$ & $\begin{array}{l}\text { Intervention - } \\
20 / 24 \\
\text { completed } \\
\text { Control - } \\
10 / 23 \\
\text { Mean } \\
\text { attendance - } \\
4.75 \text { sessions }\end{array}$ & $\begin{array}{l}\text { STAI } \\
\text { FFMQ } \\
\text { PSS } \\
\text { PRA } \\
\text { PSA }\end{array}$ & $\begin{array}{l}\text { Significant decrease in stress in } \\
\text { intervention group } \\
\text { Decrease in stress from baseline to } \\
\text { post intervention in both groups }\end{array}$ & 8 \\
\hline
\end{tabular}

BAI = Beck Anxiety Inventory; BDI-II = Beck Depression Inventory; BMSWI = Body Mind Spirit Well-being Inventory; CBSEI-C32 = Chinese Childbirth SelfEfficacy Inventory, CES-D = Centre for Epidemiologic Studies Depression Scale; CWS = Cambridge Worry Scale; DASS-D/A/S = Depression Anxiety and Stress; DES = Differential Emotional Scale; EPDS = Edinburgh Postnatal Depression Scale; ERQ = Emotional Regulation Questionnaire; FFMQ = Five Factor Mindfulness Scale; GAD 7 = General Anxiety Disorder Scale; MINI = Mini Mental State Examination; MAAS = Mindful Attention Awareness Scale; MINI = Mini International Neuropsychiatric Interview; MSSS = Maternity Social Support Scale; OWLS= Oxford Worries about Labour Scale; PANAS-X Positive and negative affect schedule extended, PAS = Pregnancy Anxiety Scale; PSA = Pregnancy Specific Anxiety; PES= Pregnancy Experience Scale; PRA = Pregnancy Related Anxiety Scale; PSOM = Positive States of Mind; PSRS = Pregnancy Stress Rating Scale; PSS = Perceived Stress Scale; PSWQ = Penn State Worry Questionnaire; SDS = Self-rating Depression Scale; SPWB = Scales of Psychological Well-Being; STAl-S = State Trait Anxiety Inventory, state sub scale., TMS=Toronto Mindfulness Scale; TPDS = Tilbury Pregnancy Distress Scale; WOC = Expanded version of Ways of coping, PHQ $-9=$ Patient Health questionnaire 


\begin{tabular}{|c|c|c|c|c|c|c|}
\hline Study & Intervention & Recruitment & Attrition & Measures & Result & CASP \\
\hline $\begin{array}{l}\text { Bowen et al (2014) } \\
\text { Canada } \\
\text { Non - randomized } \\
\text { (matched) } \\
\text { Comparative } \\
\text { study. Mixed } \\
\text { methods }\end{array}$ & $\begin{array}{l}\text { 6-week } \\
\text { interpersonal } \\
\text { group or } \\
\text { Mindfulness } \\
\text { group with some } \\
\text { focus on } \\
\text { psychoeducation }\end{array}$ & $\begin{array}{l}n=38 \text { women } 15-28 \\
\text { weeks pregnant }\end{array}$ & $\begin{array}{l}37 / 38 \\
\text { completed } \\
\text { Mean } \\
\text { attendance - } \\
4.405 \\
\text { sessions }\end{array}$ & $\begin{array}{l}\text { EPDS } \\
\text { CWS } \\
\text { STAI } \\
\text { MSSS }\end{array}$ & $\begin{array}{l}\text { No change in depression, anxiety or } \\
\text { worry between groups however a } \\
\text { significant decrease in anxiety and } \\
\text { depression was found in both groups. } \\
\text { Acceptability of groups confirmed }\end{array}$ & 7 \\
\hline $\begin{array}{l}\text { Byrne et al (2014) } \\
\text { Australia } \\
\text { Single group pre- } \\
\text { post intervention }\end{array}$ & $\begin{array}{l}8 \text { weeks } \\
2.5 \mathrm{hr} / \text { week } \\
\text { MBCE with } \\
\text { empowerment } \\
\text { model. Partners } \\
\text { attended } \\
\text { CD and workbook } \\
\text { for daily home } \\
\text { practice }\end{array}$ & $\begin{array}{l}n=12 \text { pregnant women } \\
18-28 \text { weeks and } \\
\text { partners }\end{array}$ & $\begin{array}{l}\text { 12/18 } \\
\text { completed, } \\
\text { Session } \\
\text { attendance } \\
\text { not reported }\end{array}$ & $\begin{array}{l}\text { DASS-21 } \\
\text { EPDS } \\
\text { MAAS } \\
\text { Efficacy } \\
\text { Inventory } \\
\text { Fear of } \\
\text { childbirth } \\
\text { before labour } \\
\text { Wijma } \\
\text { delivery } \\
\text { expectancy } \\
\text { questionnaire }\end{array}$ & $\begin{array}{l}\text { Significant improvement in self- } \\
\text { efficacy } \\
\text { Significant decrease in fear of birth } \\
\text { Improved Mindfulness but not } \\
\text { statistically significant } \\
\text { Decreased depression but not } \\
\text { statistically significant }\end{array}$ & 10 \\
\hline $\begin{array}{l}\text { Woolhouse et al } \\
\mathbf{2 0 1 4} \\
\text { Australia } \\
\text { Study } 1 \text { - Single } \\
\text { group pre- post } \\
\text { interventions } \\
\text { Study } 2 \text { - RCT with } \\
\text { controlled TAU } \\
\text { group }\end{array}$ & $\begin{array}{l}8 \text { week } 2 \mathrm{hr} / \text { week } \\
\text { Adapted MBSR/ } \\
\text { MBCT/MBCP } \\
\text { MindBodyBaby }\end{array}$ & $\begin{array}{l}\text { Study } 1-n=20 \text { women } \\
\text { between } 10-34 \text { weeks } \\
\text { at risk of depression, } \\
\text { stress and anxiety } \\
\text { Study } 2-n=10 \text { women } \\
\text { from universal sample } \\
\text { of }-34 \text { weeks, } 13 \text { to } \\
\text { intervention } 10 \text { to } \\
\text { control }\end{array}$ & $\begin{array}{l}11 / 20 \\
\text { completed } \\
\text { Intervention } \\
13 / 17 \\
\text { completed } \\
\text { Control- } 10 / 15 \\
\text { (Drop out in } \\
\text { both studies } \\
\text { mainly due to } \\
\text { birth }\end{array}$ & $\begin{array}{l}\text { DASS-21 } \\
\text { FFMQ } \\
\text { STAI } \\
\text { PSS } \\
\text { CES-D }\end{array}$ & $\begin{array}{l}\text { Significant improvement in } \\
\text { depression and anxiety post } \\
\text { intervention } \\
\text { Non statistically significant decrease } \\
\text { in stress } \\
\text { Significant increase in mindfulness } \\
\text { and awareness }\end{array}$ & 8 \\
\hline $\begin{array}{l}\text { Dimidjian et al } \\
\text { (2014) } \\
\text { USA } \\
\text { Single pre-post } \\
\text { intervention group } \\
\text { with qualitative } \\
\text { description }\end{array}$ & $\begin{array}{l}8 \text { sessions MBCT- } \\
\text { PD } \\
2 \mathrm{hr} / \text { week } \\
\text { Focus on PND } \\
\text { Audio files and } \\
\text { DVD provided for } \\
\text { home practice }\end{array}$ & $\begin{array}{l}n=49 \text { pregnant women } \\
\text { up to } 32 \text { weeks with } \\
\text { history of depression }\end{array}$ & $\begin{array}{l}42 / 49 \\
\text { completed }\end{array}$ & $\begin{array}{l}\text { Client } \\
\text { Satisfaction } \\
\text { Questionnaire } \\
\text { EPDS } \\
\text { (LIFE) } \\
\text { MBCT } \\
\text { Adherence } \\
\text { Scale }\end{array}$ & $\begin{array}{l}\text { Significant decrease in pregnancy } \\
\text { scores continuing through to } \\
\text { postnatal period }\end{array}$ & 10 \\
\hline
\end{tabular}

BAI = Beck Anxiety Inventory; BDI-II = Beck Depression Inventory; BMSWI = Body Mind Spirit Well-being Inventory; CBSEI-C32 = Chinese Childbirth SelfEfficacy Inventory, CES-D = Centre for Epidemiologic Studies Depression Scale; CWS = Cambridge Worry Scale; DASS-D/A/S = Depression Anxiety and Stress; DES = Differential Emotional Scale; EPDS = Edinburgh Postnatal Depression Scale; ERQ = Emotional Regulation Questionnaire; FFMQ = Five Factor Mindfulness Scale; GAD 7 = General Anxiety Disorder Scale; MINI = Mini Mental State Examination; MAAS = Mindful Attention Awareness Scale; MINI = Mini International Neuropsychiatric Interview; MSSS = Maternity Social Support Scale; OWLS= Oxford Worries about Labour Scale; PANAS-X Positive and negative affect schedule extended, PAS = Pregnancy Anxiety Scale; PSA = Pregnancy Specific Anxiety; PES= Pregnancy Experience Scale; PRA = Pregnancy Related Anxiety Scale; PSOM = Positive States of Mind; PSRS = Pregnancy Stress Rating Scale; PSS = Perceived Stress Scale; PSWQ = Penn State Worry Questionnaire; SDS = Self-rating Depression Scale; SPWB = Scales of Psychological Well-Being; STAl-S = State Trait Anxiety Inventory, state sub scale., TMS=Toronto Mindfulness Scale; TPDS = Tilbury Pregnancy Distress Scale; WOC = Expanded version of Ways of coping, PHQ $-9=$ Patient Health questionnaire 


\begin{tabular}{|c|c|c|c|c|c|c|}
\hline Study & Intervention & Recruitment & Attrition & Measures & Result & CASP \\
\hline $\begin{array}{l}\text { Zhang and Emory } \\
\text { (2015) } \\
\text { USA } \\
\text { RTC controlled } \\
\text { with TAU }\end{array}$ & $\begin{array}{l}8 \text { sessions over } 4 \\
\text { weeks } 2 \mathrm{hr} \\
\text { sessions Mindful } \\
\text { Motherhood } \\
\text { aimed at } \\
\text { improving mood } \\
\text { and infant } \\
\text { interaction }\end{array}$ & $\begin{array}{l}n=65 \text { African American } \\
\text { women } \\
31 \text { TAU and } 34 \\
\text { intervention }\end{array}$ & $\begin{array}{l}\text { Intervention - } \\
17 / 34 \\
\text { completed } \\
\text { Control - } \\
17 / 31\end{array}$ & $\begin{array}{l}\text { BD-II } \\
\text { PSS } \\
\text { TMS } \\
\text { Baseline } \\
\text { cortisol } \\
\text { Reactive } \\
\text { cortisol } \\
\text { PES } \\
\text { uplift } \\
\text { intensity } \\
\text { Pregnancy } \\
\text { Experience } \\
\text { Scale-Brief } \\
\text { Version: } \\
\text { hassle } \\
\text { intensity }\end{array}$ & $\begin{array}{l}\text { Improved mindfulness not significant } \\
\text { into post-natal period } \\
\text { No statistical significance in } \\
\text { perceived stress and baseline cortisol } \\
\text { levels } \\
\text { Improvement in pregnancy related } \\
\text { positive experience to } 1 \text { month post- } \\
\text { partum } \\
\text { Decreased depressive symptoms to } 1 \\
\text { month postpartum }\end{array}$ & 9 \\
\hline $\begin{array}{l}\text { Dimidjian et al } \\
\text { (2016) } \\
\text { USA } \\
\text { RCT with TAU }\end{array}$ & $\begin{array}{l}8 \text { sessions MBCT- } \\
\text { PD } \\
\text { 2hr/week } \\
\text { Audio files and } \\
\text { DVD provided for } \\
\text { home practice }\end{array}$ & $\begin{array}{l}n=86 \text { pregnant women } \\
\text { up to } 32 \text { weeks } \\
\text { pregnant with MDD } \\
43 \text { women to } \\
\text { interventions } 43 \text { to TAU }\end{array}$ & $\begin{array}{l}\text { Intervention } \\
-32 / 43 \\
\text { completed } \\
\text { Control - } \\
37 / 43\end{array}$ & $\begin{array}{l}\text { EPDS } \\
\text { LIFE } \\
\text { Instructor } \\
\text { Adherence } \\
\text { Scale } \\
\text { Client } \\
\text { Satisfaction } \\
\text { Questionnaire } \\
\text { Service } \\
\text { utilisation }\end{array}$ & $\begin{array}{l}\text { Higher satisfaction than control } \\
\text { Significant decrease in depression } \\
\text { severity in intervention group } \\
\text { Significant decrease in depressive } \\
\text { relapse } \\
\text { No difference in service utilisation }\end{array}$ & 9 \\
\hline $\begin{array}{l}\text { Muthukrishnan } \\
\text { et al. (2016) } \\
\text { India } \\
\text { RCT with TAU }\end{array}$ & $\begin{array}{l}\text { Mindfulness } \\
\text { meditation } 2 \\
\text { sessions/ week } \\
\text { for } 5 \text { weeks } \\
\text { CD for home } \\
\text { practice }\end{array}$ & $\begin{array}{l}n=74 \text { women } 12 \text { weeks } \\
\text { pregnant, } 34 \text { women in } \\
\text { intervention group } 40 \text { to } \\
\text { TAU (obstetric care) }\end{array}$ & Not reported & $\begin{array}{l}\text { Autonomic } \\
\text { functioning } \\
\text { tests } \\
\text { PSS }\end{array}$ & $\begin{array}{l}\text { Significant decrease in stress scores } \\
\text { Decrease in blood pressure changes } \\
\text { increase in heart rate variability }\end{array}$ & 8 \\
\hline $\begin{array}{l}\text { Yazdanimehr et al } \\
\text { (2016) } \\
\text { Iran } \\
\text { RCT with TAU }\end{array}$ & $\begin{array}{l}\text { Mindfulness } \\
\text { Integrated } \\
\text { cognitive Therapy } \\
8 \times 90 \text { min } \\
\text { sessions }\end{array}$ & $\begin{array}{l}n=80 \text { pregnant women } \\
\text { self-identifying } \\
\text { depression and anxiety } \\
n=33 \text { control } \\
n=30 \text { intervention }\end{array}$ & $\begin{array}{l}7 \text { lost to } \\
\text { control due to } \\
\text { pregnancy } \\
\text { related } \\
\text { complications } \\
10 \text { lost to } \\
\text { intervention } \\
\text { due to poor } \\
\text { attendance }\end{array}$ & $\begin{array}{l}\text { EPDS } \\
\text { BAI }\end{array}$ & $\begin{array}{l}\text { Significant decrease in depression } \\
\text { and anxiety }\end{array}$ & 10 \\
\hline $\begin{array}{l}\text { Beattie et al (2017) } \\
\text { Australia } \\
\text { Mixed methods } \\
\text { RCT pilot trial }\end{array}$ & $\begin{array}{l}8 \text { sessions } \\
\text { 2hr/week Drawing } \\
\text { on MBCT, MBPC } \\
\text { and Mindful } \\
\text { Motherhood } \\
\text { (Vieten and Astin, } \\
\text { 2008) } \\
\text { Home practice }\end{array}$ & $\begin{array}{l}n=48 \text { women } \\
\text { randomized to } \\
\text { mindfulness based or } \\
\text { pregnancy support } \\
\text { program. Exclusion of } \\
\text { score higher than } 13 \text { on } \\
\text { EPDS or identifying self- } \\
\text { harm ideation }\end{array}$ & $\begin{array}{l}\text { 17/23 } \\
\text { completed }\end{array}$ & $\begin{array}{l}\text { PSS } \\
\text { EPDS } \\
\text { MAAS } \\
\text { Birth } \\
\text { Outcomes }\end{array}$ & $\begin{array}{l}\text { No statistically significant difference } \\
\text { between groups, improved self- } \\
\text { awareness in both groups decreasing } \\
\text { self-reported stress. } \\
\text { Feasibility and acceptability of } \\
\text { intervention confirmed }\end{array}$ & 8 \\
\hline
\end{tabular}

BAI = Beck Anxiety Inventory; BDI-II = Beck Depression Inventory; BMSWI = Body Mind Spirit Well-being Inventory; CBSEI-C32 = Chinese Childbirth SelfEfficacy Inventory, CES-D = Centre for Epidemiologic Studies Depression Scale; CWS = Cambridge Worry Scale; DASS-D/A/S = Depression Anxiety and Stress; DES = Differential Emotional Scale; EPDS = Edinburgh Postnatal Depression Scale; ERQ = Emotional Regulation Questionnaire; FFMQ = Five Factor Mindfulness Scale; GAD 7 = General Anxiety Disorder Scale; MINI = Mini Mental State Examination; MAAS = Mindful Attention Awareness Scale; MINI = Mini International Neuropsychiatric Interview; MSSS = Maternity Social Support Scale; OWLS= Oxford Worries about Labour Scale; PANAS-X Positive and negative affect schedule extended, PAS = Pregnancy Anxiety Scale; PSA = Pregnancy Specific Anxiety; PES= Pregnancy Experience Scale; PRA = Pregnancy Related Anxiety Scale; PSOM = Positive States of Mind; PSRS = Pregnancy Stress Rating Scale; PSS = Perceived Stress Scale; PSWQ = Penn State Worry Questionnaire; SDS = Self-rating Depression Scale; SPWB = Scales of Psychological Well-Being; STAl-S = State Trait Anxiety Inventory, state sub scale., TMS=Toronto Mindfulness Scale; TPDS = Tilbury Pregnancy Distress Scale; WOC = Expanded version of Ways of coping, PHQ $-9=$ Patient Health questionnaire 


\begin{tabular}{|c|c|c|c|c|c|c|}
\hline Study & Intervention & Recruitment & Attrition & Measures & Result & CASP \\
\hline \multirow{9}{*}{$\begin{array}{l}\text { Townshend et al } \\
\text { (2018) } \\
\text { Australia } \\
\text { Single group Pre } \\
\text { and post } \\
\text { intervention } \\
\text { Data collected } \\
\text { from pre-existing } \\
\text { dataset }\end{array}$} & 8 weeks & \multirow{9}{*}{$\begin{array}{l}n=109 \text { pregnant women } \\
\text { with history or current } \\
\text { depression }\end{array}$} & \multirow{9}{*}{$\begin{array}{l}\text { Sample of } \\
109 \\
\text { Attendance } \\
\text { and } \\
\text { completion } \\
\text { rates not } \\
\text { reported } \\
\text { although } \\
\text { states high } \\
\text { attrition. }\end{array}$} & EPDS & Significant reduction in depression, & 6 \\
\hline & \multirow[t]{5}{*}{ Adapted MBCT } & & & DASS-21 & & \\
\hline & & & & & $\begin{array}{l}\text { Improved mindfulness and self- } \\
\text { compassion }\end{array}$ & \\
\hline & & & & & & \\
\hline & & & & & & \\
\hline & & & & & & \\
\hline & \multirow{3}{*}{$\begin{array}{l}30 \text { mind daily } \\
\text { home practice }\end{array}$} & & & PASS & & \\
\hline & & & & MAAS & & \\
\hline & & & & FFMQ & & \\
\hline \multirow{7}{*}{$\begin{array}{l}\text { Warriner et al } \\
\text { (2018) } \\
\text { United Kingdom } \\
\text { Single group Pre } \\
\text { and post } \\
\text { intervention }\end{array}$} & \multirow{2}{*}{$\begin{array}{l}4 \text { weeks, 2,5hr } \\
\text { sessions }\end{array}$} & \multirow{2}{*}{$\begin{array}{l}155 \text { individuals ( } 86 \\
\text { women and } 69 \text { men) }\end{array}$} & \multirow{7}{*}{$\begin{array}{l}\mathrm{n}=36 \text { women } \\
\text { completed pre } \\
\text { and post } \\
\text { intervention } \\
\text { data } \\
\text { collection }\end{array}$} & EPDS & Significant improvements in stress, & 7 \\
\hline & & & & FFMQ & & \\
\hline & \multirow{5}{*}{ Adapted MBPC } & \multirow{5}{*}{$\begin{array}{l}\text { Data only collected for } \\
\text { women for this review }\end{array}$} & & PSS & & \\
\hline & & & & GAD-7 & & \\
\hline & & & & TPDS & & \\
\hline & & & & OWLS & & \\
\hline & & & & PES & & \\
\hline \multirow{5}{*}{$\begin{array}{l}\text { Lönnberg et al. } \\
\text { (2019) } \\
\text { Sweden } \\
\text { RCT with } \\
\text { controlled Lamaze } \\
\text { group }\end{array}$} & \multirow{5}{*}{$\begin{array}{l}8 \text { weeks and } \\
\text { reunion } \\
\text { Adapted MBCP }\end{array}$} & \multirow{5}{*}{$\begin{array}{l}n=197 \text { pregnant } \\
\text { women with a history of } \\
\text { depression, anxiety, } \\
\text { early life adversity and } \\
\text { current high levels of } \\
\text { perceived stress } \\
\text { intervention } n=96 \\
\text { control } n=97\end{array}$} & \multirow{5}{*}{$\begin{array}{l}\text { Intervention } \\
-11 \text { lost to } \\
\text { follow up } \\
\text { Control - } 5 \\
\text { lost to follow } \\
\text { up }\end{array}$} & PSS & \multirow{5}{*}{$\begin{array}{l}\text { Compared to the active control } \\
\text { treatment, MBCP significantly } \\
\text { reduced perceived stress }(p=0.038, d \\
=0.30) \text { and depressive symptoms }(p \\
=0.004, d=0.42) \text {, and increased } \\
\text { positive states of mind }(p=0.005, d= \\
0.41) \text { and self-reported mindfulness } \\
(p=0.039, d=0.30) \text {. }\end{array}$} & \multirow[t]{5}{*}{11} \\
\hline & & & & EPDS & & \\
\hline & & & & PSOM & & \\
\hline & & & & FFMQ & & \\
\hline & & & & & & \\
\hline \multirow{2}{*}{$\begin{array}{l}\text { Pan et al. } \\
(2019)\end{array}$} & \multirow{2}{*}{$\begin{array}{l}8 \text { weeks of } 3 \mathrm{hr} \\
\text { sessions and } \\
\text { reunion }\end{array}$} & \multirow[t]{4}{*}{$n=104$ pregnant women } & \multirow{4}{*}{$\begin{array}{l}8 \text { lost to } \\
\text { follow up }\end{array}$} & PPS & \multirow{4}{*}{$\begin{array}{l}\text { Improvement found in both groups } \\
\text { but relatively more improvement in } \\
\text { the intervention group }\end{array}$} & \multirow[t]{4}{*}{12} \\
\hline & & & & EPDS & & \\
\hline Taiwan & MBCP & & & FFMQ & & \\
\hline RCT with TAU & $\begin{array}{l}\text { Practice at home } \\
\text { audio recordings }\end{array}$ & & & CBSEI-C32 & & \\
\hline \multirow{2}{*}{$\begin{array}{l}\text { Zhang et al. } \\
\text { (2019) }\end{array}$} & \multirow{3}{*}{$\begin{array}{l}8 \text { week } 90 \text { min } \\
\text { MBSR }\end{array}$} & $n=63$ & \multirow{3}{*}{$\begin{array}{l}3 \text { lost to } \\
\text { follow up }\end{array}$} & STAI & \multirow{4}{*}{$\begin{array}{l}\text { Significant decrease in stress and } \\
\text { anxiety. No significant difference in } \\
\text { depression although baseline } \\
\text { depression scores were low }\end{array}$} & \multirow[t]{4}{*}{11} \\
\hline & & \multirow[t]{3}{*}{ intervention $n=34$} & & PSRS & & \\
\hline China & & & & SDS & & \\
\hline RCT with TAU & & & & & & \\
\hline
\end{tabular}

BAI = Beck Anxiety Inventory; BDI-II = Beck Depression Inventory; BMSWI = Body Mind Spirit Well-being Inventory; CBSEI-C32 = Chinese Childbirth SelfEfficacy Inventory, CES-D = Centre for Epidemiologic Studies Depression Scale; CWS = Cambridge Worry Scale; DASS-D/A/S = Depression Anxiety and Stress; DES = Differential Emotional Scale; EPDS = Edinburgh Postnatal Depression Scale; ERQ = Emotional Regulation Questionnaire; FFMQ = Five Factor Mindfulness Scale; GAD 7 = General Anxiety Disorder Scale; MINI = Mini Mental State Examination; MAAS = Mindful Attention Awareness Scale; MINI = Mini International Neuropsychiatric Interview; MSSS = Maternity Social Support Scale; OWLS= Oxford Worries about Labour Scale; PANAS-X Positive and negative affect schedule extended, PAS = Pregnancy Anxiety Scale; PSA = Pregnancy Specific Anxiety; PES= Pregnancy Experience Scale; PRA = Pregnancy Related Anxiety Scale; PSOM = Positive States of Mind; PSRS = Pregnancy Stress Rating Scale; PSS = Perceived Stress Scale; PSWQ = Penn State Worry Questionnaire; SDS = Self-rating Depression Scale; SPWB = Scales of Psychological Well-Being; STAl-S = State Trait Anxiety Inventory, state sub scale., TMS=Toronto Mindfulness Scale; TPDS = Tilbury Pregnancy Distress Scale; WOC = Expanded version of Ways of coping, PHQ $-9=$ Patient Health questionnaire 


\begin{tabular}{|c|c|c|c|c|c|c|}
\hline Study & Intervention & Recruitment & Attrition & Measures & Result & CASP \\
\hline \multirow{5}{*}{$\begin{array}{l}\text { Zemestani and } \\
\text { Nikoo } \\
\text { (2020) } \\
\text { Iran } \\
\text { RCT with } \\
\text { psychoeducatione } \\
\text { sessions }\end{array}$} & \multirow{5}{*}{$\begin{array}{l}8 \text { week } 2 \mathrm{hr} \\
\text { sessions MBCT } \\
\text { 30min daily home } \\
\text { practice }\end{array}$} & \multirow{6}{*}{$\begin{array}{l}n=38 \text { pregnant women } \\
\text { with depression and } \\
\text { comorbid anxiety }\end{array}$} & \multirow{6}{*}{$\begin{array}{l}1 \text { lost to } \\
\text { follow up }\end{array}$} & BDI-II & \multirow{6}{*}{$\begin{array}{l}\text { Significant improvement in } \\
\text { depression and anxiety from control } \\
\text { group and over time }\end{array}$} & \multirow[t]{6}{*}{10} \\
\hline & & & & BAI & & \\
\hline & & & & ERQ & & \\
\hline & & & & & & \\
\hline & & & & & & \\
\hline \multicolumn{3}{|c|}{ SPWB } & & & & \\
\hline $\begin{array}{l}\text { BAI = Beck Anxie } \\
\text { Efficacy Inventor } \\
\text { Stress; DES = Dif } \\
\text { Mindfulness Sca } \\
\text { Mini Internationa } \\
\text { negative affect s } \\
\text { Pregnancy Relat } \\
\text { State Worry Que } \\
\text { sub scale., TMS } \\
\text { Health questionr }\end{array}$ & $\begin{array}{l}\text { ventory; BDI-II = Beck } \\
\text { S-D = Centre for Epid } \\
\text { ntial Emotional Scale } \\
\text { AD } 7=\text { General Anxie } \\
\text { uropsychiatric Intervi } \\
\text { dule extended, PAS = } \\
\text { nxiety Scale; PSOM = } \\
\text { naire; SDS = Self-rati } \\
\text { onto Mindfulness Sca }\end{array}$ & $\begin{array}{l}\text { Depression Inventory; BMS } \\
\text { miologic Studies Depressi } \\
\text { EPDS = Edinburgh Postna } \\
\text { y Disorder Scale; MINI = M } \\
\text { w; MSSS = Maternity Soci } \\
\text { regnancy Anxiety Scale; PS } \\
\text { gositive States of Mind; PS } \\
\text { g Depression Scale; SPWB } \\
\text { e; TPDS = Tilbury Pregnan }\end{array}$ & $\begin{array}{l}\mathrm{VI}=\text { Body Mi } \\
\text { n Scale; CW } \\
\text { al Depressior } \\
\text { i Mental Sta } \\
\text { Support Sc } \\
\text { A = Pregnan } \\
\text { RS = Pregnar } \\
\text { = Scales of P } \\
\text { y Distress S }\end{array}$ & $\begin{array}{l}\text { irit Well-being } \\
\text { Imbridge Wor } \\
\text { e; ERQ = Emo } \\
\text { amination; M } \\
\text { NLS= Oxford } \\
\text { ecific Anxiety } \\
\text { ress Rating S } \\
\text { ological Well- } \\
\text { VOC = Expanc }\end{array}$ & $\begin{array}{l}\text { entory; CBSEI-C32 = Chinese Childbirth } \\
\text { cale; DASS-D/A/S = Depression Anxiety } \\
\text { al Regulation Questionnaire; FFMQ = Fi } \\
=\text { Rindful Attention Awareness Scale; } \\
\text { ries about Labour Scale; PANAS-X Posit } \\
\text { = Pregnancy Experience Scale; PRA = } \\
\text { PSS = Perceived Stress Scale; PSWQ = } \\
\text { g; STAI-S = State Trait Anxiety Inventory } \\
\text { ersion of Ways of coping, PHQ -9= Pati }\end{array}$ & $\begin{array}{l}\mathrm{f}- \\
\text { id } \\
\text { Factor } \\
\mathrm{Nl}= \\
\text { and } \\
\text { enn } \\
\text { tate } \\
\mathrm{t}\end{array}$ \\
\hline
\end{tabular}




\begin{tabular}{|c|c|c|c|c|c|c|c|c|c|c|c|c|}
\hline & \multicolumn{12}{|c|}{ Randomised Control Trials } \\
\hline & $\begin{array}{l}\text { Vieten } \\
\text { and } \\
\text { Astin }\end{array}$ & $\begin{array}{l}\text { Guardino } \\
\text { et al }\end{array}$ & $\begin{array}{l}\text { Woolhouse } \\
\text { et al Study } \\
2\end{array}$ & $\begin{array}{l}\text { Zhang } \\
\text { and } \\
\text { Emory }\end{array}$ & $\begin{array}{l}\text { Dimidjian } \\
\text { et al }\end{array}$ & $\begin{array}{l}\text { Muthukrishnan } \\
\text { et al }\end{array}$ & $\begin{array}{l}\text { Yazdanimehr } \\
\text { et al }\end{array}$ & $\begin{array}{l}\text { Beattie } \\
\text { et al }\end{array}$ & $\begin{array}{l}\text { Lönnberg } \\
\text { et al }\end{array}$ & $\begin{array}{l}\text { Pan } \\
\text { et } \\
\text { al. }\end{array}$ & $\begin{array}{l}\text { Zhang } \\
\text { et al }\end{array}$ & $\bar{z}$ \\
\hline 1. Aim? & 1 & 1 & 1 & 1 & 1 & 1 & 1 & 1 & 1 & 1 & 1 & 1 \\
\hline $\begin{array}{l}\text { 2.Participants } \\
\text { randomised? }\end{array}$ & 1 & 1 & 1 & 1 & 1 & 1 & 1 & 1 & 1 & 1 & 1 & 1 \\
\hline $\begin{array}{l}\text { 3. Were all } \\
\text { participants } \\
\text { accounted for? }\end{array}$ & 1 & 1 & 1 & 0 & 0 & $?$ & 1 & 0 & 1 & 1 & 1 & 1 \\
\hline $\begin{array}{l}\text { 4a.Participants } \\
\text { blind to }\end{array}$ & 0 & 0 & 0 & 0 & 0 & 0 & 0 & 0 & 0 & 0 & 0 & $c$ \\
\hline intervention? & 0 & 0 & 0 & 0 & 0 & 0 & 0 & 0 & 1 & 1 & 1 & $c$ \\
\hline $\begin{array}{l}\text { 4b.Investigator } \\
\text { blind to } \\
\text { interventions? }\end{array}$ & 0 & 0 & 0 & 0 & 0 & 1 & $?$ & 0 & 0 & 1 & 1 & 1 \\
\hline \multicolumn{13}{|l|}{$\begin{array}{l}\text { 4c. Analysers } \\
\text { blind? }\end{array}$} \\
\hline $\begin{array}{l}\text { 5. Were the } \\
\text { study groups } \\
\text { similar? }\end{array}$ & 1 & 1 & 0 & 1 & 1 & $?$ & 1 & 1 & 1 & 1 & 1 & 1 \\
\hline $\begin{array}{l}\text { 6. Did each } \\
\text { study group } \\
\text { receive same } \\
\text { level of care }\end{array}$ & 1 & 0 & 1 & 1 & 1 & 1 & 1 & 0 & 0 & 1 & 1 & 1 \\
\hline $\begin{array}{l}\text { 7. Results } \\
\text { comprehensive? }\end{array}$ & 1 & 1 & 1 & 1 & 1 & 1 & 1 & 1 & 1 & 1 & 1 & 1 \\
\hline $\begin{array}{l}\text { 8. Was precision } \\
\text { treatment effect } \\
\text { reported? }\end{array}$ & 0 & 1 & 1 & 1 & 1 & 1 & 1 & 1 & 1 & 1 & 1 & 1 \\
\hline $\begin{array}{l}\text { 9.Do benefits } \\
\text { outweigh the } \\
\text { harms }\end{array}$ & 1 & 1 & 1 & 1 & 1 & 1 & 1 & 1 & 1 & 1 & 1 & 1 \\
\hline 10a.Can results & 1 & 1 & 1 & 1 & 1 & 1 & 1 & 1 & 1 & 1 & 1 & 1 \\
\hline locally & 1 & 1 & 1 & 1 & 1 & 1 & 1 & 1 & 1 & 1 & 1 & 1 \\
\hline $\begin{array}{l}\text { 10b.Would } \\
\text { intervention } \\
\text { provide greater } \\
\text { value than } \\
\text { existing } \\
\text { intervention? }\end{array}$ & & & & & & & & & & & & \\
\hline $1=\mathrm{YES} 0=\mathrm{NO} ?$ & JNSURE & & & & & & & & & & & \\
\hline
\end{tabular}


Table 3

Appraisal of the articles, CASP Cohort Studies

\begin{tabular}{|c|c|c|c|c|c|c|c|}
\hline & $\begin{array}{l}\text { Duncan and } \\
\text { Bardacke } \\
\text { (2009) }\end{array}$ & $\begin{array}{l}\text { Goodman } \\
\text { et al } \\
\text { (2013) }\end{array}$ & $\begin{array}{l}\text { Byme et } \\
\text { al } \\
(2014)\end{array}$ & $\begin{array}{l}\text { Woolhouse } \\
\text { et al (2014) }\end{array}$ & $\begin{array}{l}\text { Dimidjian } \\
\text { et al } \\
\text { (2014) }\end{array}$ & $\begin{array}{l}\text { Townshend } \\
\text { et al (2018) }\end{array}$ & $\begin{array}{l}\text { Warriner } \\
\text { et al } \\
\text { (2018) }\end{array}$ \\
\hline & USA & USA & Australia & Study 1 & USA & & UK \\
\hline 1.Did the study address a clearly focused issue? & 1 & 1 & 1 & 1 & 1 & 1 & 1 \\
\hline $\begin{array}{l}\text { 2. Was the cohort recruited in an acceptable } \\
\text { way? }\end{array}$ & 0 & 1 & 1 & 1 & 1 & 0 & 0 \\
\hline $\begin{array}{l}\text { 3.Was the exposure accurately measured to } \\
\text { minimise bias? }\end{array}$ & 1 & 1 & 1 & 1 & 1 & 1 & 1 \\
\hline $\begin{array}{l}\text { 4. Was the outcome accurately measured to } \\
\text { minimise bias? }\end{array}$ & 1 & 1 & 1 & 1 & 1 & 0 & 1 \\
\hline \multirow{2}{*}{$\begin{array}{l}\text { 5a. Have the authors identified all important } \\
\text { confounding factors? } \\
\text { 5b.Have they taken account of the confounding } \\
\text { factors in the design and/or analysis? }\end{array}$} & 1 & 1 & 1 & 1 & 1 & 1 & 1 \\
\hline & 1 & 1 & 1 & 1 & 1 & 1 & 1 \\
\hline \multirow{2}{*}{$\begin{array}{l}\text { 6a.Was the follow up of subjects complete } \\
\text { enough? } \\
\text { 6b.Was the follow up of subjects long enough? }\end{array}$} & 1 & 1 & 1 & 0 & 1 & 0 & 0 \\
\hline & 1 & 1 & 1 & 0 & 1 & 0 & 0 \\
\hline $\begin{array}{l}\text { 7. Can the results be applied to the local } \\
\text { population? }\end{array}$ & 1 & 1 & 1 & 1 & 1 & 1 & 1 \\
\hline $\begin{array}{l}\text { 8. Do the results of this study fit with other } \\
\text { available evidence? }\end{array}$ & 1 & 1 & 1 & 1 & 1 & 1 & 1 \\
\hline $1=$ YES $0=$ NO $?=$ UNSURE & & & & & & & \\
\hline
\end{tabular}

Due to the heterogeneity between studies in terms of methodology, interventions and recruitment, a narrative synthesis was conducted. The interpretation and synthesis of information considers the differences between studies where research designs are not comparable (40). Consistent with the scope of the review, the findings have been explored within the domains of perinatal depression, anxiety, and stress.

\section{Results}

The initial search yielded 2053 articles, which resulted in 1986 records following duplicate removal. Following title and abstract review, and with additional hand searching, 54 reports were assessed via a full-text review. 20 studies remained eligible for inclusion from 21 participant cohorts (see figure 1 ). One study (28) reported results from two cohorts hereafter labelled study 1 and 2. Studies were excluded due to several reasons including: mixed intervention including Mindfulness based yoga (41, 42, 43), ACT (44), self-compassion (31) and behaviour change therapy (45), samples selected based upon specific physical conditions such as overeating (46); psychological conditions such as bipolar spectrum disorder (46); extreme fear of childbirth (48), studies that included participants who were not yet pregnant but trying to conceive or adopting (49) and online interventions were excluded (50). A summary of study characteristics and results is presented in Table 1.

\section{Study characteristics}

All studies included quantitative data and seven included qualitative outcomes. Of the included studies twelve were randomised controlled trials (RCTs) (51, $52,28$, study $2 ; 53,54,55,56,57,58,59,60,61)$, two studies were non-randomised $(62,63)$ and eight were non-controlled $(23,28,64,65$, Study $1,66,67,68)$. The RCTs used varying methods of control including a wait list group (51), a reading group (52) and treatment as usual $(28,53,54,55,50,51)$, a pregnancy support group (57), Lamaze group (58) and psychoeducational sessions (61).

Participant recruitment was predominantly from hospitals and obstetric clinics. All eligible studies involved a sample of pregnant women; however, one study involved the intervention being undertaken in a community setting mixed with the general population (52). Three studies involved partners, although no data is included in this review on non- pregnant participants $(3,65,68)$. One study recruited women based on a self- identified 'mood disturbance' $(51)$; eight studies recruited women with a history of anxiety and/or depression the remaining studies recruited a universal sample of generally healthy pregnant women. Other characteristics that were reported on included age of participants, gestation and parity, and marital and education status.

Most of the studies were undertaken in either the United States of America or Australia, one study came from Canada (63) one from India (55), one from Sweden $(58)$, two from Taiwan $(59,60)$ and two from Iran $(56,61)$. The gestation of participants was between $12-28$ weeks with Dimidjian and colleagues (2015) accepting participants until 32 weeks. All studies describe most participants as primiparous except Zhang and Emory (2015) who described $84.6 \%$ of participants already having children. Participants were over 18 years in all studies with an average age between $28-34$ years. Most studies identified participants to be married and having secondary education. Only one study specifically recruited from a low socio-economic, minority (African American) cohort (53). 
All eligible studies involved a MBI in a group setting and ranged from 4 - 9 weeks in duration with

each session 2 -3 hours in duration. The feasibility study by Warriner and colleagues (2018) acknowledged that the length of some studies, particularly the Mindfulness Based Childbirth and Parenting (MBCP) interventions (24 hours) may not be feasible within a health care setting and effect adoption. All the studies except Muthukrishnan et al (2016) and Yazdanimehr et al (2016) involved 'homework' which was generally described as mindfulness practices sometimes aided by a recording or workbook and logged on a time sheet. A wide range of adaptations to the traditional MBSR and MBCT were found. Fifteen of the studies identified as having a basis in MBSR and/or MBCT and were adapted to the perinatal period; six of the studies did not identify a basis in MBSR or MBCT. Most interventions included a component of psychoeducation alongside the development of mindfulness skills. Two studies focused on the improvement of mood $(51,53)$, eight focused on depression $(28,54,56,58,61,62,66)$, one on anxiety reduction (64) and five were adapted to include childbirth education and mindful parenting $(23,28,58,65,68)$. The remaining studies aimed to improve general well-being. The study by Zhang and Emory (2015) had such high attrition rates (3 out of 34 randomized participants completed 8 sessions) that dose-effect analyses were used to test the treatment effect on outcome variables.

All the studies collected pre- and post-intervention data with some studies collecting post-partum data varying between 3 weeks and 6 months post-partum. Outcome measures varied between studies. All studies used self-report measures to determine emotional health and mindfulness which may indicate some reporting bias. One study measured salivary cortisol measures (53) and one study looked at autonomic functioning tests (55). Townsend et al. (2018) collected data from a pre-existing hospital dataset for women who attended the intervention between 2010 -2016, the instruments used were reported to have changed over this time. All the studies used validated measurement tools, however due to the significant variety of outcome tools and measured mental health outcomes it was difficult to compare the effect of the interventions. Inconsistent effect was found across the reviewed studies. All studies included multiple components such as psychological support, mindfulness practice, discussion, parent education and peer support, however outcome measures were only reported on mental health and mindfulness. None of the studies demonstrated quantitative measures on the therapeutic mechanism of peer and/or facilitator support. Outcomes for depression, anxiety, stress, and mindfulness are reported here, other outcomes can be found in Table 4. 
Table 4

Outcomes of Studies

\begin{tabular}{|c|c|c|c|c|c|c|c|}
\hline Study & Depression & Anxiety & Stress & Mindfulness & Other & Qualitative & Post-natal \\
\hline $\begin{array}{l}\text { Vieten and } \\
\text { Astin (2008) }\end{array}$ & $\begin{array}{l}\text { Non-statistically } \\
\text { significant } \\
\text { improvement }\end{array}$ & $\begin{array}{l}\text { Significant } \\
\text { improvement }\end{array}$ & $\begin{array}{l}\text { Non-statistically } \\
\text { significant } \\
\text { improvement }\end{array}$ & $\begin{array}{l}\text { Non- } \\
\text { statistically } \\
\text { significant } \\
\text { improvement }\end{array}$ & $\begin{array}{l}\text { Significantly } \\
\text { reduced } \\
\text { negative affect }\end{array}$ & $\mathrm{N} / \mathrm{A}$ & $\begin{array}{l}\text { Non } \\
\text { statistically } \\
\text { significant } \\
\text { improvement } \\
\text { at } 3 \text { months } \\
\text { post-partum }\end{array}$ \\
\hline $\begin{array}{l}\text { Duncan and } \\
\text { Bardacke } \\
\text { (2009) }\end{array}$ & \multirow[t]{2}{*}{$\begin{array}{l}\text { Improvement with } \\
\text { small effect size }\end{array}$} & \multirow[t]{2}{*}{$\begin{array}{l}\text { Significant } \\
\text { improvement }\end{array}$} & \multirow[t]{2}{*}{$\begin{array}{l}\text { Statistically } \\
\text { significant } \\
\text { decrease in } \\
\text { stress }\end{array}$} & \multirow[t]{2}{*}{$\begin{array}{l}\text { Significant } \\
\text { improvement }\end{array}$} & \multirow[t]{2}{*}{$\begin{array}{l}\text { Minimal } \\
\text { change to } \\
\text { positive or } \\
\text { negative affect }\end{array}$} & $\begin{array}{l}\text { Participants } \\
\text { continuing to use } \\
\text { skills to post- } \\
\text { partum. }\end{array}$ & \multirow[t]{2}{*}{ Not measured } \\
\hline USA & & & & & & $\begin{array}{l}\text { Supported by } \\
\text { group }\end{array}$ & \\
\hline $\begin{array}{l}\text { Dunn et al } \\
(2012) \\
\text { Australia }\end{array}$ & $\begin{array}{l}\text { Improvement in } 50 \% \\
\text { of treatment group }\end{array}$ & $\begin{array}{l}\text { Non- } \\
\text { statistically } \\
\text { significant } \\
\text { improvement }\end{array}$ & $\begin{array}{l}75 \% \text { decrease in } \\
\text { stress lasting } \\
\text { into postnatal } \\
\text { period }\end{array}$ & $\begin{array}{l}\text { Non- } \\
\text { statistically } \\
\text { significant } \\
\text { improvement }\end{array}$ & $\begin{array}{l}\text { Self- } \\
\text { compassion - } \\
67 \% \text { of } \\
\text { treatment } \\
\text { group reported } \\
\text { improvement }\end{array}$ & $\begin{array}{l}\text { Mindfulness } \\
\text { practiced beyond } \\
\text { completion of } \\
\text { intervention. } \\
\text { Living in the } \\
\text { moment and } \\
\text { acceptance. }\end{array}$ & $\begin{array}{l}\text { Improvements } \\
\text { in measures } \\
\text { at } 6 \text { weeks } \\
\text { post-partum }\end{array}$ \\
\hline $\begin{array}{l}\text { Goodman et al } \\
(2013) \\
\text { USA }\end{array}$ & $\begin{array}{l}\text { Statistically } \\
\text { significant } \\
\text { improvement }\end{array}$ & $\begin{array}{l}\text { Statistically } \\
\text { significant } \\
\text { improvement }\end{array}$ & Not measured & $\begin{array}{l}\text { Statistically } \\
\text { significant } \\
\text { improvement }\end{array}$ & $\begin{array}{l}\text { Statistically } \\
\text { significant } \\
\text { improvement } \\
\text { in worry }\end{array}$ & $\begin{array}{l}\text { Recommendations } \\
\text { include ongoing } \\
\text { support, inclusion } \\
\text { of partners, } \\
\text { decrease } \\
\text { homework }\end{array}$ & Not measured \\
\hline $\begin{array}{l}\text { Guardino et al } \\
(2013) \\
\text { USA }\end{array}$ & Not measured & $\begin{array}{l}\text { Significant } \\
\text { decrease in } \\
\text { anxiety in } \\
\text { comparison } \\
\text { with control }\end{array}$ & $\begin{array}{l}\text { Significant } \\
\text { decrease in } \\
\text { stress in both } \\
\text { intervention and } \\
\text { control group }\end{array}$ & $\begin{array}{l}\text { Significant } \\
\text { increase in } \\
\text { mindfulness } \\
\text { in both } \\
\text { groups }\end{array}$ & $\mathrm{N} / \mathrm{A}$ & $\mathrm{N} / \mathrm{A}$ & $\begin{array}{l}\text { Improvements } \\
\text { not } \\
\text { maintained at } \\
6 \text { weeks post- } \\
\text { partum }\end{array}$ \\
\hline $\begin{array}{l}\text { Bowen et al } \\
\text { (2014) } \\
\text { Canada }\end{array}$ & $\begin{array}{l}\text { No difference } \\
\text { between groups }\end{array}$ & $\begin{array}{l}\text { Significant } \\
\text { decrease in } \\
\text { anxiety for } \\
\text { both groups } \\
\text { from pre- } \\
\text { intervention to } \\
\text { post-partum }\end{array}$ & Not measured & $\begin{array}{l}\text { Not } \\
\text { measured }\end{array}$ & $\begin{array}{l}\text { Worry - No } \\
\text { difference } \\
\text { between } \\
\text { groups }\end{array}$ & $\begin{array}{l}\text { High level of } \\
\text { acceptability of } \\
\text { group }\end{array}$ & $\begin{array}{l}\text { Improvements } \\
\text { in both } \\
\text { groups } \\
\text { continue at } 4 \\
\text { weeks post- } \\
\text { partum }\end{array}$ \\
\hline $\begin{array}{l}\text { Byrne et al } \\
(2014) \\
\text { Australia }\end{array}$ & $\begin{array}{l}\text { Non-statistically } \\
\text { significant } \\
\text { improvement }\end{array}$ & $\begin{array}{l}\text { Non- } \\
\text { statistically } \\
\text { significant } \\
\text { improvement }\end{array}$ & Minimal effect & $\begin{array}{l}\text { Non- } \\
\text { statistically } \\
\text { significant } \\
\text { improvement }\end{array}$ & $\begin{array}{l}\text { Self-efficacy } \\
\text { and birth } \\
\text { expectation } \\
\text { significantly } \\
\text { improved, } \\
\text { decreased fear } \\
\text { of childbirth }\end{array}$ & $\begin{array}{l}\text { Increased sense of } \\
\text { community and } \\
\text { empowerment }\end{array}$ & $\begin{array}{l}3-12 \text { weeks } \\
\text { post-partum }\end{array}$ \\
\hline $\begin{array}{l}\text { Woolhouse et } \\
\text { al } 2014 \\
\text { Australia (I) }\end{array}$ & $\begin{array}{l}\text { Statistically } \\
\text { significant } \\
\text { improvement }\end{array}$ & $\begin{array}{l}\text { Statistically } \\
\text { significant } \\
\text { improvement }\end{array}$ & $\begin{array}{l}\text { Non-statistically } \\
\text { significant } \\
\text { improvement }\end{array}$ & $\begin{array}{l}\text { Statistically } \\
\text { significant } \\
\text { improvement } \\
\text { in } 2 \text { of } 5 \\
\text { measures }\end{array}$ & $\mathrm{N} / \mathrm{A}$ & $\begin{array}{l}\text { Increased ability to } \\
\text { manage negativity, } \\
\text { Improved } \\
\text { relationships } \\
\text { Improved sleep } \\
\text { Improved quality } \\
\text { of life }\end{array}$ & Not measured \\
\hline $\begin{array}{l}\text { Woolhouse et } \\
\text { al } 2014 \\
\text { Australia (II) }\end{array}$ & $\begin{array}{l}\text { Statistically } \\
\text { significant } \\
\text { improvement for } \\
\text { both intervention and } \\
\text { control }\end{array}$ & $\begin{array}{l}\text { Statistically } \\
\text { significant } \\
\text { improvement } \\
\text { for both } \\
\text { intervention } \\
\text { and control }\end{array}$ & $\begin{array}{l}\text { Statistically } \\
\text { significant } \\
\text { improvement for } \\
\text { both intervention } \\
\text { and control }\end{array}$ & $\begin{array}{l}\text { Statistically } \\
\text { significant } \\
\text { improvement } \\
\text { for both } \\
\text { intervention } \\
\text { and control }\end{array}$ & $\begin{array}{l}\text { Statistically } \\
\text { significant } \\
\text { improvement } \\
\text { for both } \\
\text { intervention } \\
\text { and control }\end{array}$ & N/A & Not measured \\
\hline $\begin{array}{l}\text { Dimidjian et al } \\
\text { (2014) }\end{array}$ & $\begin{array}{l}\text { Significant decrease } \\
\text { in depression relapse }\end{array}$ & Not measured & Not measured & $\begin{array}{l}\text { Not } \\
\text { measured }\end{array}$ & $\begin{array}{l}\text { High degree of } \\
\text { satisfaction in } \\
\text { intervention } \\
\text { reported }\end{array}$ & $\mathrm{N} / \mathrm{A}$ & $\begin{array}{l}\text { Decreased } \\
\text { depressive } \\
\text { symptoms } \\
\text { and relapse at } \\
\text { 1st and 6th } \\
\text { months }\end{array}$ \\
\hline $\begin{array}{l}\text { Zhang and } \\
\text { Emory (2015) } \\
\text { USA }\end{array}$ & $\begin{array}{l}\text { Medium effect size } \\
\text { for number of } \\
\text { mindfulness } \\
\text { sessions attended } \\
\text { and levels of } \\
\text { mindfulness at post } \\
\text { intervention }\end{array}$ & Not measured & $\begin{array}{l}\text { No effect on } \\
\text { perceived stress }\end{array}$ & $\begin{array}{l}\text { Positive } \\
\text { effect not } \\
\text { lasting into } \\
\text { postnatal } \\
\text { period }\end{array}$ & $\begin{array}{l}\text { Session } \\
\text { numbers had } \\
\text { large effect on } \\
\text { reactive } \\
\text { cortisol level }\end{array}$ & $\mathrm{N} / \mathrm{A}$ & $\begin{array}{l}\text { No difference } \\
\text { between } \\
\text { groups at 1- } \\
\text { month post- } \\
\text { partum }\end{array}$ \\
\hline
\end{tabular}




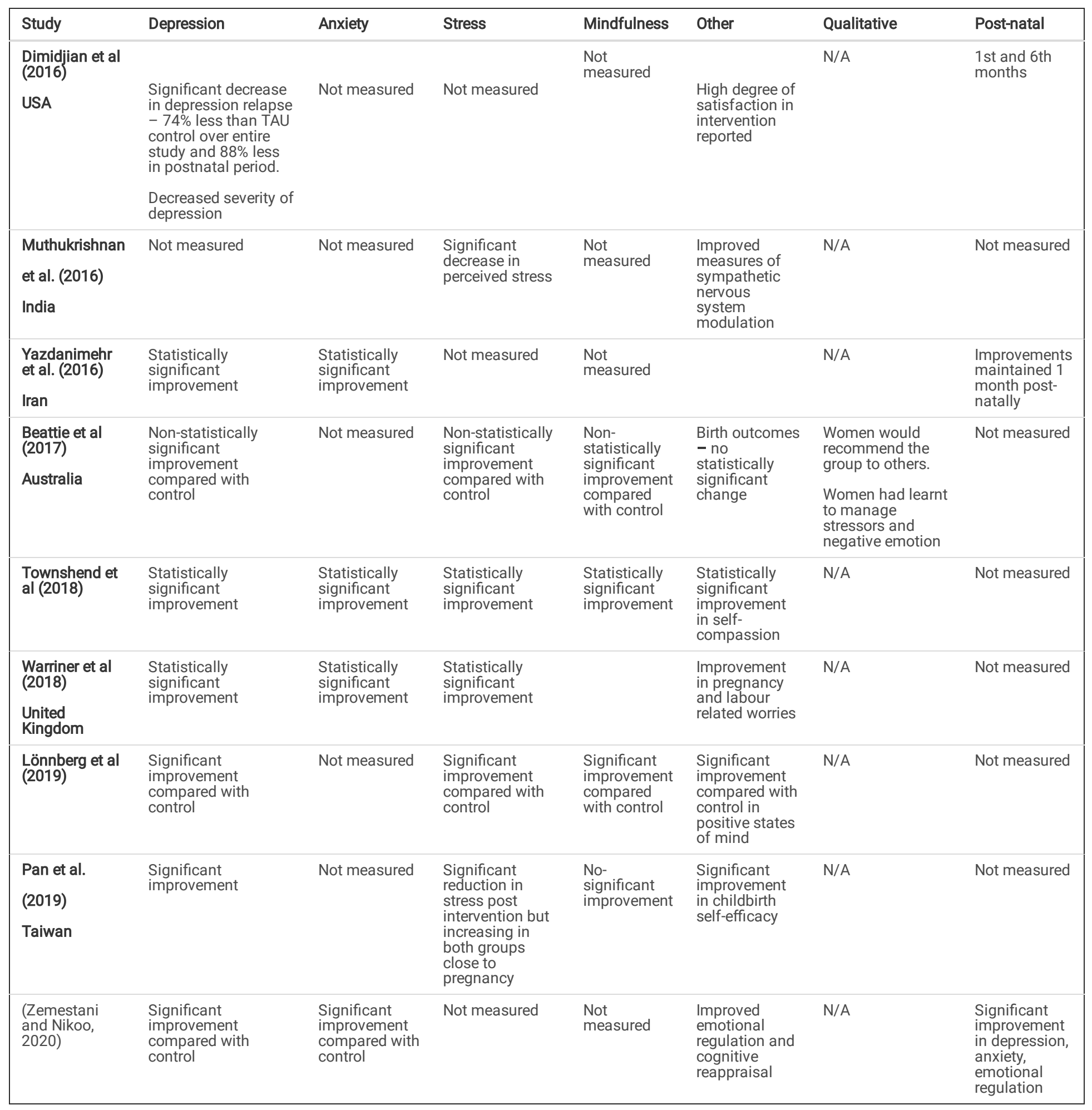

\section{Depression, Anxiety and Perinatal Stress}

Depression was measured as an outcome in all but two studies with the Edinburgh Postnatal Depression Scale (EPDS), but also included the depression arm of the Depression Anxiety and Stress Scale (DASS-21) and others. Outcomes of depression in the RCTs was variable. Yazdanimehr et al. (2016); Lönnberg et al. (2019) and Zemestani and Nikoo (2020) had statistically significant improvement in depression compared to control group and Dimidjian et al. 2015 (MBCT) found significant decrease in depressive relapse that was maintained into the post-natal period. Several studies found a decrease in depressive symptoms that was not statistically significant however the data suggested a post intervention improvement $(28,51)$. Beattie et al. (2017) and Zhang and Emory (2015) found no difference between intervention and control post intervention, however Zhang and Emory (2015) found a decrease in depressive symptoms at 1-month post-partum related to the amount of sessions attended. Dunn et al.'s (2012) non-controlled intervention found half of the women in the treatment group had an improvement in depressive symptoms. In the single group interventions, $(23,28$ Study $1,64,66,67,68)$ most found significant improvement in depression; however, Byrne et al. (2014) found a non-significant difference in depression scores. Bowen et al.'s (2014) comparative study 
found a decrease in depressive symptoms however no statistically significant difference was found between groups. Outcomes for depression show some evidence of treatment effect however this was less significant in studies comparing MBls to control groups.

The effectiveness of the MBI on anxiety levels was measured in fourteen of the eligible studies. Measuring tools for anxiety were varied and measured both general anxiety and pregnancy specific anxiety, measuring tools included the anxiety arm of the DASS-21, Beck Anxiety Inventory (BAI), General Anxiety Disorder Scale (GAD - 7), Pregnancy Specific Anxiety (PSA); Pregnancy Experience Scale (PES); Pregnancy Related Anxiety Scale (PRA). Among the RCTs that measured anxiety, all found a reduction in anxiety scores compared to control group (28 study $2,51,52,56,60,61,67)$. The non-randomized study (62) found a non-statistically significant improvement, and the non-controlled studies all found significant improvement in anxiety scores $(23,28,64,65,68)$. Bowen et al., (2014) comparative study found a decrease in anxiety in both groups with a non-significant difference between groups. Studies with statistically significant improvements in anxiety also tended to show statistically significant improvements in mindfulness.

Guardino et al. (2014), Muthukrishnan et al. (2016), Lönnberg et al. (2019) and Zhang et al. (2019) demonstrated a statistically significant decrease in pre to post intervention perceived stress scores as well as improved stress related autonomic functioning scores in the Muthukrishnan et al. (2016) study. Vieten and Astin (2008) and Beattie et al. (2017) reported a non-statistically significant improvement due to small effect size whilst Zhang and Emory (2015) found no effect in stress scores in relation to how many intervention sessions were attended. Woolhouse et al. (2012) study 2 and Guardino et al. (2014) reported a statistically significant decrease in stress scores for both the intervention and control groups. Dunn et al. (2012) found $75 \%$ of participants in the intervention group reported a decrease in stress scores into the post-natal period. Among the single group studies that reported stress outcomes Duncan and Bardacke (2009), Townsend et al. (2018) and Warriner et al. (2018) reported statistically significant improvements in stress measures while Woolhouse et al. (2012) study 2 reported a non-statistically significant improvement and Byrne et al. (2012) reported minimal effect on stress scores.

\section{Mindfulness}

Pre to post intervention changes in mindfulness were measured in most studies, among the RCTs that measured mindfulness, Vieten and Astin (2008) and Lönnberg et al. (2019) reported a significant increase in self-reported Mindfulness. Beattie et al. (2017) reported a non-significant increase compared to control and both Guardino et al. (2014) and Woolhouse et al. (2012) study 2 reported a statistically significant increase in mindfulness scores for both the intervention and control groups. Dunn et al. (2012) found a non-statistically significant improvement in mindfulness. The non-controlled studies showed statistically significant mindfulness scores in all studies except Byrne et al. (2012) who found a non-significant improvement in mindfulness measures.

\section{Qualitative Findings}

Seven of the twenty-one studies included qualitative data. Feedback was generally positive and demonstrated acceptability of the intervention. Themes that emerged from interviews included skill development and continued use of new skills, connection, and suggestions for improvement.

Skills learnt included an increased ability to stay in the present moment, acceptance of whatever is arising (57) and enhanced empowerment to cope with difficult situations (65). The ability to reign in negative patterns (28) and release stress (57) was also described. Several studies noted that women were continuing to apply these skills during labour and in the immediate post-partum period $(23,65)$. This also applied to studies that did not have a childbirth and parenting education focus (63). Participants described continuing to practice formal and informal mindful techniques after the completion of the groups; in one study this was also expressed by women who did not complete the intervention (62).

Participants generally described the group as supportive and suggested that forming relationships, having experience normalised through shared stories (62) and development of community was a positive experience. In one study (28) several participants described an initial feeling of discomfort in the first session when the group had not yet bonded although this improved throughout the intervention.

Suggested improvements included partner participation so that partners would be able to support women with the skills they had learnt (57, 64) and ongoing support with mindfulness techniques $(57,64)$. The participants in Byrne et al $(2018)$ study suggested both increased ongoing support but also that the length of sessions could be reduced. Homework was described as helpful but sometimes felt like 'too much' (64). Responding to women's views and experiences will help to inform the design of interventions which are acceptable to women and to develop an understanding of how and why intervention components may contribute to outcomes.

\section{Discussion}

Research into the effects of Mindfulness-based Interventions in pregnancy is in early stages and the current research is lacking in rigorous methodology and consistency in intervention and outcome measures. Of the 21 studies eligible, 11 were RCTs several of which were arguably underpowered and statistically significant difference was not found. Of the RCTs, the control groups varied and included wait list groups, treatment as usual, reading, support group, childbirth or psychological education. As such, all participants in control groups continued to receive health service support and education, with four of the RCTs demonstrating outcome improvements in both the intervention and control ( 28 Study $2,52,57,63)$. It was suggested that the control-groups within these studies may have provided some benefit through peer support in pregnancy. Information received in the control groups may have also provided some benefit such as reading material (52), pregnancy support group (57) and Interpersonal group therapy (63). In one RCT up to $50 \%$ of the control group were also found to be attending practices with similar therapeutic mechanisms such yoga and meditation practice during the intervention (28) making it difficult to determine if changes in data was in response to the intervention alone. Guardino et al., (2013) also reported 30\% of the control group were attending an antenatal yoga group during the intervention; this may have provided a similar focus thus decreasing the difference in effect between groups. There is also a possibility that women who accept participating in research on mental health interventions may have a greater awareness and motivation toward improving mental health outcomes regardless of their randomization. 
A great variety in the format or focus of MBls was found; with differing content, intervention length and follow up. Several studies included a childbirth and parenting component $(23,28$ Study 1 and $2,53,57,58)$ and others focussed on mental health education $(51,56,61,63,64,65)$. Selection criteria also varied between universal populations of pregnant women to women recruited who identified as having mood concerns (51), high anxiety (28 Study, 52,64$)$ and/or depression (28 Study, 54, 56, 64, 65, 66). Beattie et al (2017) excluded participants with an EPDS of over 13 . This may account for some of the underpowered changes to depression scores as the self-reported depression scores were low prior to intervention.

According to Taylor et al's (2016) meta-analysis of the effectiveness of MBls in the perinatal population, the pre and post effect size of depression and anxiety were increased when the intervention recruited from a population of pregnant women vulnerable to anxiety and depression. This was demonstrated particularly in the interventions that offered a psycho-education component to the intervention and may indicate increased motivation among these participants. One study reported a higher drop-out rate in participants with lower mental health scores and suggested this may be due to these participants feeling like they did not require the tools taught in this style of intervention (68).

The self-reporting assessment tools that were used to determine the mental health outcomes were diverse, as were the time frames in which they were performed, making comparison difficult. Several studies did not study all outcomes. Almost all the measured outcomes in the selected interventions demonstrated some improvement in the self-reported outcomes, however due to under-powered sample sizes the statistical significance of the results were reduced. The studies with the larger sample sizes showed greater statistically significant improvements in both mental health and mindfulness outcomes (54, $55,56,58,67)$. Most of the studies reviewed pre and post intervention changes in mindfulness as this was the hypothesized therapeutic mechanism of change. Lönnberg et al (2019) explored the mediating effect of mindfulness in their robust study and found that changes in aspects of mindfulness outcomes had significant mediating effect on the changes in the outcomes of depression and perceived stress. A correlation between significantly decreased depressive symptoms and increased mindfulness was observed in several studies (28 study 1, 64, 67).

Length of intervention was also diverse and ranged from 4 to 10 weeks with differing periods of engagement with instructors and recommended 'homework' periods. Optimal length of intervention an MBI was not confirmed (69). Several studies have suggested the intervention length may prevent some participants from participating if it is deemed too long and may be a greater burden on the health system (69). Overall a high level of acceptability of MBIs in pregnancy were reported. Attendance was high in all groups apart from the Zhang and Emory (2015) study which recruited from a cohort of low income women, although no reason was given for the high attrition rates in this study, it does highlight the need for continued research around supporting the mental health of socially vulnerable women during pregnancy.

Demographic variation was limited in the reviewed studies. Participants were primarily well educated, married and nulliparous women. Despite research demonstrating that women from low socio-economic, ethnic backgrounds may be at higher risk of mental health concerns (70) only one study recruited from a population that identified as a low-socioeconomic ethnic group (53). Due to growing multiculturalism and the differences that may occur in the cultural expressions of emotion it is valuable to understand the acceptability and efficacy of these interventions in a variety of cultures (61). This systematic review demonstrated greater cultural variations than previous reviews of this topic, also signifying that MBls are being applied in a diverse range of cultures and health settings. The lack of multiparous recruitment may indicate that attendance of interventions is more difficult due to issues with childcare, this could be explored in future research. It was also noted that although all eligible studies were group interventions, none of the literature applied measured outcomes to identify how the group support influenced the study. Three of the controlled studies $(57,63)$ found improvements in mental health outcomes in both the intervention and control groups possibly suggesting that peer support may have a mediating effect in these outcomes. Qualitative data demonstrated that the group support was beneficial $(23,65)$. Group support has been found to be beneficial in both mental health studies and in the pregnant population through pregnancy support and childbirth education groups $(34,63)$. Although the detrimental effects of perinatal mental health concerns on the infant and parent/infant interaction has been broadly researched and the benefit of mindfulness on parenting has been studied, none of the reviewed studies included a measure of this. Townshend et al. (2018) adjusted their intervention to include elements of attachment theory and Lönnberg et al. (2019) included teaching methods to increase awareness of the baby, however no measure of the effect of this was demonstrated nor long-term follow up studied.

\section{Conclusion}

This review found that Mindfulness-based group interventions afford modest improvements in perinatal depression, anxiety, stress, and mindfulness during pregnancy; however, study size was globally small and transferability of findings thus limited. There was also a lack of consistency in studies, which contributes to difficulty with result interpretation. Further research is needed. Future studies that focused on mental health education and outcomes for women experiencing mental health difficulties would be of benefit. While more robust methodology including comparison with a randomised control is recommended, caution in choosing control method is advised for future research. Future studies addressing the specific obstacles to attendance that women of difficult social situations face, would be of value. Reduced length of intervention should be explored as it may be more acceptable to women with diverse social situation and potentially more cost effective for health systems. It would also be of value to explore how group support affected any change mechanisms within the participants. This review highlighted the need for future research that incorporates maternal/fetal bonding into the intervention and measured outcomes.

\section{Declarations}

\section{Ethics Approval and consent to participate}

Not Applicable

\section{Consent for publication}


Not Applicable

\section{Availability of data and materials}

Not Applicable

\section{Author Contributions}

AJ Conceptualisation, Methodology, validation, formal analysis, writing draft, review and editing

LK Conceptualisation, Methodology, validation, formal analysis, writing draft, review and editing, supervision

KG Conceptualisation, Methodology, validation, formal analysis, writing draft, review and editing, supervision

LK-D Conceptualisation, Methodology, validation, formal analysis, writing draft, review and editing, supervision

\section{Acknowledgement}

This material has not been published in whole or in part elsewhere; the manuscript is not currently being considered for publication in another journal; and, all authors have been personally and actively involved in substantive work leading to the manuscript and will hold themselves jointly and individually responsible for its content.

\section{Competing interest}

The authors declare that they have no competing interests

\section{Funding}

No direct or indirect funding supported this study.

\section{Ethical statement}

This material has not been published in whole or in part elsewhere; the manuscript is not currently being considered for publication in another journal; and, all authors have been personally and actively involved in substantive work leading to the manuscript and will hold themselves jointly and individually responsible for its content.

\section{Conflict of interest}

None declared.

\section{References}

1. Australian Institute of Health and Welfare 2020. Maternal deaths in Australia. Cat. no. PER 99. Canberra: AlHW. Viewed 20 May 2021, https://www.aihw.gov.au/reports/mothers-babies/maternal-deaths-in-australia

2. Lewis, A. J., Austin, E., Knapp, R., Vaiano, T., \& Galbally, M. Perinatal Maternal Mental Health, Fetal Programming and Child Development. Healthcare (Basel, Switzerland). 2015; 3(4), 1212-1227. https://doi.org/10.3390/healthcare3041212

3. Dagher R, Bruckheim H, Colpe L, Edwards E, White D. Perinatal Depression: Challenges and Opportunities. Journal of Women's Health. 2021;30(2):154159.

4. Australian Institute of Health and Welfare. Australia's mothers and babies 2016-in brief. 2018. Perinatal statistics series no. 34. Cat. no. PER 97. Canberra: AlHW.

5. Szegda K, Markenson G, Bertone-Johnson E, Chasan-Taber L. Depression during pregnancy: a risk factor for adverse neonatal outcomes? A critical review of the literature. The Journal of Maternal-Fetal \& Neonatal Medicine. 2013;27(9):960-967.

6. Felder J, Laraia B, Coleman-Phox K, Bush N, Suresh M, Thomas M et al. Poor Sleep Quality, Psychological Distress, and the Buffering Effect of Mindfulness Training During Pregnancy. Behavioral Sleep Medicine. 2017;16(6):611-624.

7. Byrn M, Penckofer S. The Relationship Between Gestational Diabetes and Antenatal Depression. Journal of Obstetric, Gynecologic \& Neonatal Nursing. 2015;44(2):246-255.

8. Pawlby S, Sharp D, Hay D, O'Keane V. Antenatal depression predicts depression in adolescent offspring: Prospective longitudinal community-based study. Journal of Affective Disorders. 2008;107:S54-S55.

9. Skouteris, H., Wertheim, E., Rallis, S., Milgrom, J. and Paxton, S.. Depression and anxiety through pregnancy and the early postpartum: An examination of prospective relationships. Journal of Affective Disorders. 2009; 113(3), pp.303-308.

10. Misri, S., Abizadeh, J., Sanders, S. and Swift, E. Perinatal Generalized Anxiety Disorder: Assessment and Treatment. 2015. Journal of Women's Health, 24(9), pp.762-770

11. Dunkel Schetter, C. and Tanner, L.. Anxiety, depression and stress in pregnancy. Current Opinion in Psychiatry. 2012; 25(2), pp.141-148. 
12. Isgut, M., Smith, A., Reimann, E., Kucuk, O. and Ryan, J.. The impact of psychological distress during pregnancy on the developing fetus: biological mechanisms and the potential benefits of mindfulness interventions. 2017; Journal of Perinatal Medicine, 45(9).

13. Rubertsson, C., Hellström, J., Cross, M. and Sydsjö, G.. Anxiety in early pregnancy: prevalence and contributing factors. Archives of Women's Mental Health. 2014; 17(3), pp.221-228.

14. Bowlby, J 1988b, A Secure Base, Basic Books, New York.

15. Stone, S., Diop, H., Declercq, E., Cabral, H., Fox, M. and Wise, L. Stressful Events During Pregnancy and Postpartum Depressive Symptoms. Journal of Women's Health. 2015; 24(5), pp.384-393.

16. Yelland, J., Sutherland, G. and Brown, S. Postpartum anxiety, depression and social health: findings from a population-based survey of Australian women. BMC Public Health. 2010; 10(1).

17. Isgut M, Smith A, Reimann E, Kucuk O, Ryan J. The impact of psychological distress during pregnancy on the developing fetus: biological mechanisms and the potential benefits of mindfulness interventions. Journal of Perinatal Medicine. 2017; 45(9).

18. Bayrampour, H., Hapsari, A. P., \& Pavlovic, J.. Barriers to addressing perinatal mental health issues in midwifery settings. Midwifery. 2018; 59 , 47-58.

19. Sandall, J., Soltani, H., Gates, S., Shennan, A. and Devane, D. Midwife-led continuity models versus other models of care for childbearing women. 2016; Cochrane Database of Systematic Reviews.

20. Kabat-Zinn, J. Wherever you go, there you are: Mindfulness meditation in everyday life. New York. 1994; Hyperion.

21. Kabat-Zinn, J.. Mindfulness-based interventions in context: past, present, and future. Clinical Psychology: Science and Practice. 2003; $10(2), 144-156$.

22. Segal, Z. V., Williams, J. M. G., \& Teasdale, J. D. Preventing depression: mindfulness-based cognitive therapy. 2002; New York: Guilford.

23. Duncan, L. and Bardacke, N.. Mindfulness-Based Childbirth and Parenting Education: Promoting Family Mindfulness During the Perinatal Period. Journal of Child and Family Studies. 2009; 19(2), pp.190-202.

24. Sundquist, J., Palmér, K., Johansson, L. and Sundquist, K. The effect of mindfulness group therapy on a broad range of psychiatric symptoms: A randomised controlled trial in primary health care. European Psychiatry. 2017; 43, pp.19-27.

25. Taylor, B. L., Cavanagh, K., \& Strauss, C.. The effectiveness of mindfulness-based interventions in the perinatal period: A systematic review and metaanalysis. Plos One. 2016; 11(5), e0155720.

26. Dhillon, A., Sparkes, E., \& Duarte, R. V.. Mindfulness-Based Interventions During Pregnancy: A Systematic Review and Meta-analysis. Mindfulness. 2017; 8(6), 1421-1437. doi:10.1007/s12671-017-0726-x

27. Shi, Z. and MacBeth, A. The Effectiveness of Mindfulness-Based Interventions on Maternal Perinatal Mental Health Outcomes: a Systematic Review. Mindfulness. 2017; 8(4), pp.823-847.

28. Woolhouse, H., Mercuri, K., Judd, F., \& Brown, S. J. Antenatal mindfulness intervention to reduce depression, anxiety and stress: a pilot randomized controlled trial of the MindBabyBody program in an Australian tertiary maternity hospital. BMC Pregnancy and Childbirth. $2014 ; 14,369-397$.

29. Braeken, M. A., Jones, A., Otte, R. A., Nyklíček, I., \& Bergh, B. R. Potential benefits of mindfulness during pregnancy on maternal autonomic nervous system function and infant development. Psychophysiology.2016; 54(2), 279-288. doi:10.1111/psyp.12782

30. Veringa, I., de Bruin, E., Bardacke, N., Duncan, L., van Steensel, F., Dirksen, C. and Bögels, S. 'I've Changed My Mind', Mindfulness-Based Childbirth and Parenting (MBCP) for pregnant women with a high level of fear of childbirth and their partners: study protocol of the quasi-experimental controlled trial. BMC Psychiatry. 2016; 16(1).

31. Perez-Blasco, J., Viguer, P. and Rodrigo, M. Effects of a mindfulness-based intervention on psychological distress, well-being, and maternal self-efficacy in breast-feeding mothers: results of a pilot study. Archives of Women's Mental Health. 2013; 16(3), pp.227-236.

32. Townshend, K., Jordan, Z., Stephenson, M. and Tsey, K. The effectiveness of mindful parenting programs in promoting parents' and children's wellbeing. JBI Database of Systematic Reviews and Implementation Reports. 2016; 14(3), pp.139-180.

33. Repper, J. and Carter, T. A review of the literature on peer support in mental health services. Journal of Mental Health. 2011; 20(4), pp.392-411.

34. Ingram, J. A mixed methods evaluation of peer support in Bristol, UK: mothers', midwives' and peer supporters' views and the effects on breastfeeding. BMC Pregnancy and Childbirth. 2013; 13(1). https://doi.org/10.1186/1471-2393-13-192

35. Dennis, C. Peer support within a health care context: A concept analysis. International Journal of Nursing Studies. 2003; 40(3), 321-332. doi:10.1016/s0020-7489(02)00092-5 https://www.researchgate.net/publication/10883370

36. Davidson, L., Chinman, M., Sells, D., \& Rowe, M. Peer Support Among Adults With Serious Mental Illness: A Report From the Field. Schizophrenia Bulletin. 2005; 32(3), 443-450. doi:10.1093/schbul/sbj043

37. Jones, C. C., Jomeen, J., \& Hayter, M. The impact of peer support in the context of perinatal mental illness: a meta-ethnography. Midwifery. 2014; 30(5), 491-498.

38. Balaji, A. B., Claussen, A. H., Smith, D. C., Visser, S. N., Morales, M. J., \& Perou, R. Social Support Networks and Maternal Mental Health and Well-Being. Journal of Womens Health. 2007; 16(10), 1386-1396. doi:10.1089/jwh.2007.cdc10

39. Moher D, Liberati A, Tetzlaff J, Altman DG, The PRISMA Group Preferred Reporting Items for Systematic Reviews and Meta-Analyses: The PRISMA Statement. PLoS Med. 2009; 6(7): e1000097. https://doi.org/10.1371/journal.pmed.1000097

40. Green, B. N., Johnson, C. D., \& Adams, A. Writing narrative literature reviews for peer-reviewed journals: Secrets of the trade. Journal of Chiropractic Medicine. 2006; 5(3), 101-117. doi: 10.1016/S0899-3467(07)60142-6

41. Beddoe, A., Paul Yang, C., Kennedy, H., Weiss, S. and Lee, K. The Effects of Mindfulness-Based Yoga During Pregnancy on Maternal Psychological and Physical Distress. Journal of Obstetric, Gynecologic \& Neonatal Nursing. 2009; 38(3), pp.310-319. 
42. Muzik, M., Hamilton, S., Lisa Rosenblum, K., Waxler, E. and Hadi, Z. Mindfulness yoga during pregnancy for psychiatrically at-risk women: Preliminary results from a pilot feasibility study. Complementary Therapies in Clinical Practice. 2012; 18(4), pp.235-240.

43. Kinser PA, Thacker LR, Rider A, Moyer S, Amstadter AB, Mazzeo SE, Bodnar-Deren S, Starkweather A. Feasibility, Acceptability, and Preliminary Effects of "Mindful Moms": A Mindful Physical Activity Intervention for Pregnant Women with Depression. Nurs Res. 2021; Mar-Apr 01;70(2):95-105. doi: 10.1097/NNR.0000000000000485. PMID: 33630532; PMCID: PMC8366589. Copy

44. Bonacquisti A, Cohen MJ, Schiller CE. Acceptance and commitment therapy for perinatal mood and anxiety disorders: development of an inpatient group intervention. Arch Womens Ment Health. 2017; Oct;20(5):645-654. doi: 10.1007/s00737-017-0735-8. Epub 2017 Jun 10. PMID: 28600645.

45. Hennelly, S.E, Perman-Howe, P, Foxcroft,D.L, Smith, L.A. The feasibility of 'Mind the Bump': A mindfulness based maternal behaviour change intervention, Complementary Therapies in Clinical Practice, Volume 40, 2020; 101178, ISSN 1744-3881, https://doi.org/10.1016/j.ctcp.2020.101178.

46. Laraia, B., Adler, N., Coleman-Phox, K., Vieten, C., Mellin, L., Kristeller, J., Thomas, M., Stotland, N., Lustig, R., Dallman, M., Hecht, F., Bush, N., de Groat, C. and Epel, E. Novel Interventions to Reduce Stress and Overeating in Overweight Pregnant Women: A Feasibility Study. Maternal and Child Health Journal. 2018; 22(5), pp.670-678.

47. Miklowitz DJ, Semple RJ, Hauser M, Elkun D, Weintraub MJ, Dimidjian S. Mindfulness-based cognitive therapy for perinatal women with depression or bipolar spectrum disorder. Cognit Ther Res. 2015;39(5):590-600.

48. Veringa IK, de Bruin El, Bardacke N, Duncan LG, van Steensel FJA, Dirksen CD, et al. "I've Changed My Mind", Mindfulness-Based Childbirth and Parenting (MBCP) for pregnant women with a high level of fear of childbirth and their partners: study protocol of the quasi-experimental controlled trial. BMC Psychiatry. 2016; 16(1):377.

49. Gambrel, L. and Piercy, F. Mindfulness-Based Relationship Education For Couples Expecting Their First Child-Part 1: A Randomized Mixed-Methods Program Evaluation. Journal of Marital and Family Therapy. 2014; 41(1), pp.5-24.

50. 50. Goetz M, Schiele C, Müller M, Matthies LM, Deutsch TM, Spano C, Graf J, Zipfel S, Bauer A, Brucker SY, Wallwiener M, Wallwiener S. Effects of a Brief Electronic Mindfulness-Based Intervention on Relieving Prenatal Depression and Anxiety in Hospitalized High-Risk Pregnant Women: Exploratory Pilot Study J Med Internet Res 2020; 22(8):e17593

51. Vieten, C., \& Astin, J. Effects of a mindfulness-based intervention during pregnancy on prenatal stress and mood: results of a pilot study. Archives of Women's Mental Health. 2008; 11(1), 67-74.

52. Guardino, C., Dunkel Schetter, C., Bower, J., Lu, M. and Smalley, S. Randomised controlled pilot trial of mindfulness training for stress reduction during pregnancy. Psychology \& Health. 2013; 29(3), pp.334-349.

53. Zhang,H.,\& Emory, E. K. A mindfulness-based intervention for pregnant African-American women. Mindfulness. 2015; 6(3), 663-674.

54. Dimidjian, S., Goodman, S., Felder, J., Gallop, R., Brown, A. and Beck, A. Staying well during pregnancy and the postpartum: A pilot randomized trial of mindfulness-based cognitive therapy for the prevention of depressive relapse/recurrence. Journal of Consulting and Clinical Psychology. 2016; 84(2), pp.134-145.

55. Muthukrishnan S, Jain R, Kohli S, Batra S. Effect of Mindfulness Meditation on Perceived Stress Scores and Autonomic Function Tests of Pregnant Indian Women. J Clin Diagn Res. 2016;10(4):CC05-CC8. doi:10.7860/JCDR/2016/16463.7679

56. Yazdanimehr, R., Omidi, A., Sadat, Z., \& Akbari, H. The Effect of Mindfulness-integrated Cognitive Behavior Therapy on Depression and Anxiety among Pregnant Women: a Randomized Clinical Trial. Journal of caring sciences. 2016; 5(3), 195-204. https://doi.org/10.15171/jcs.2016.021

57. Beattie, J., Hall, H., Biro, M., East, C. and Lau, R. Effects of mindfulness on maternal stress, depressive symptoms and awareness of present moment experience: A pilot randomised trial. Midwifery. 2017; 50, pp.174-183.

58. Lönnberg, G., Jonas, W., Unternaehrer, E., Bränström, R., Nissen, E., \& Niemi, M. Effects of a mindfulness based childbirth and parenting program on pregnant women's perceived stress and risk of perinatal depression-Results from a randomized controlled trial. Journal of affective disorders. 2020; 262, 133-142. https://doi.org/10.1016/j.jad.2019.10.048

59. Wan-Lin Pan, Meei-Ling Gau, Tzu-Ying Lee, Hei-Jen Jou, Chieh-Yu Liu, Tzung-Kuen Wen, Mindfulness-based programme on the psychological health of pregnant women, Women and Birth. 2019; Volume 32, Issue 1, , Pages e102-e109, ISSN 1871-5192, https://doi.org/10.1016/j.wombi.2018.04.018.

60. Jia-Yuan Zhang, Yu-Xia Cui, Yu-Qiu Zhou \& Ying-Li Li. Effects of mindfulness-based stress reduction on prenatal stress, anxiety and depression, Psychology, Health \& Medicine. 2019; 24:1, 51-58, DOI: 10.1080/13548506.2018.1468028

61. Zemestani, M., \& Nikoo, Z. F. Effectiveness of mindfulness-based cognitive therapy for comorbid depression and anxiety in pregnancy: A randomized controlled trial. Archives of Women's Mental Health.2019; 23, 207-214. https://doi.org/10.1007/s00737-019-00962-8.

62. Dunn, C., Hanieh, E., Roberts, R. and Powrie, R. Mindful pregnancy and childbirth: effects of a mindfulness-based intervention on women's psychological distress and well-being in the perinatal period. Archives of Women's Mental Health. 2012; 15(2), pp.139-143.

63. Bowen, A., Baetz, L., Balbeuna, L., \& Muhajarine, N. EPA-0064 - Antenatal therapy decreases depression and worry symptoms. European Psychiatry. 2014; 29, 1. doi:10.1016/s0924-9338(14)77564-9

64. Goodman, J., Guarino, A., Chenausky, K., Klein, L., Prager, J., Petersen, R., Forget, A. and Freeman, M. CALM Pregnancy: results of a pilot study of mindfulness-based cognitive therapy for perinatal anxiety. Archives of Women's Mental Health. 2014; 17(5), pp.373-387.

65. Byrne J, e. Effectiveness of a Mindfulness-Based Childbirth Education pilot study on maternal self-efficacy and fear of childbirth. PubMed. 2018; https://www.ncbi.nlm.nih.gov/pubmed/24325752/

66. Dimidjian, S., Goodman, S.H., Felder, J.N. et al. An open trial of mindfulness-based cognitive therapy for the prevention of perinatal depressive relapse/recurrence. Arch Womens Ment Health. 2015; 18,85-94. https://doi.org/10.1007/s00737-014-0468-x

Page $19 / 20$ 
67. Townshend, K., Caltabiano, N., Powrie, R. and O'Grady, H. A Preliminary Study Investigating the Effectiveness of the Caring for Body and Mind in Pregnancy (CBMP) in Reducing Perinatal Depression, Anxiety and Stress. Journal of Child and Family Studies. 2018; 27(5), pp.1556-1566.

68. Warriner, S., Crane, C., Dymond, M., Krusche, A. An evaluation of mindfulness-based childbirth and parenting courses for pregnant women and prospective fathers/partners within the UK NHS (MBCP-4-NHS), Midwifery. 2018; Volume 64, Pages 1-10, ISSN 0266-6138, https://doi.org/10.1016/j.midw.2018.05.004

69. Carmody, J., \& Baer, R.. How long does a mindfulness-based stress reduction program need to be? A review of class contact hours and effect sizes for psychological distress. Journal Of Clinical Psychology. 2009; 65(6), 627-638. doi: 10.1002/jclp.20555

70. Shakeel, N., Eberhard-Gran, M ., Sletner, L., Slinning, K., Martinsen, E., Holme, I. and Jenum, A. A prospective cohort study of depression in pregnancy, prevalence and risk factors in a multi-ethnic population. BMC Pregnancy and Childbirth. 2015; 15(1).

\section{Figures}

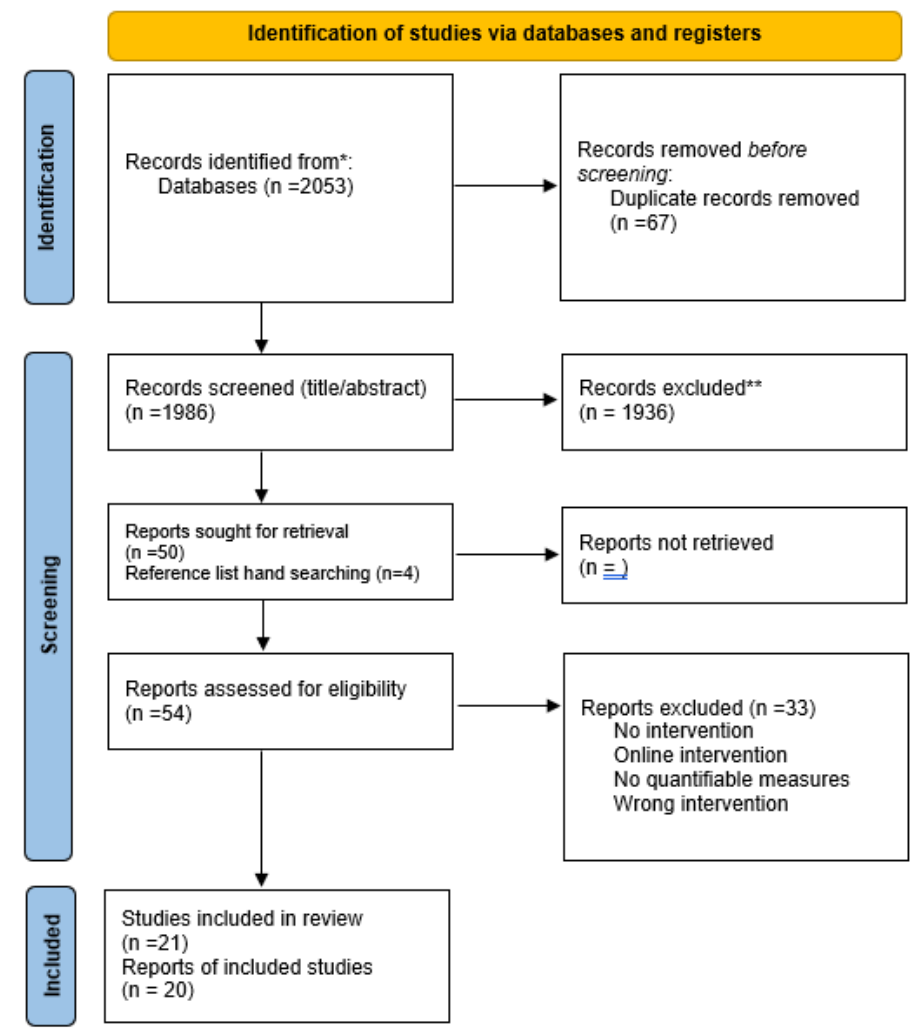

Figure 1

PRISMA flow chart 